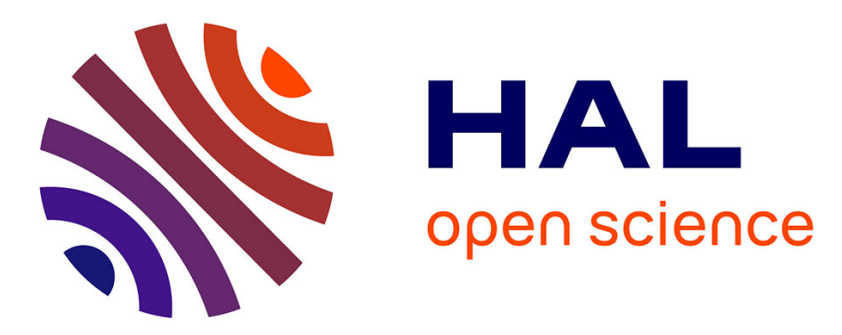

\title{
The onset of unsteadiness of two-dimensional bodies falling or rising freely in a viscous fluid: a linear study
}

Pauline Assemat, David Fabre, Jacques Magnaudet

\section{To cite this version:}

Pauline Assemat, David Fabre, Jacques Magnaudet. The onset of unsteadiness of two-dimensional bodies falling or rising freely in a viscous fluid: a linear study. Journal of Fluid Mechanics, 2012, vol. 690, pp. 173-202. 10.1017/jfm.2011.419 . hal-00908868

\section{HAL Id: hal-00908868 https://hal.science/hal-00908868}

Submitted on 25 Nov 2013

HAL is a multi-disciplinary open access archive for the deposit and dissemination of scientific research documents, whether they are published or not. The documents may come from teaching and research institutions in France or abroad, or from public or private research centers.
L'archive ouverte pluridisciplinaire HAL, est destinée au dépôt et à la diffusion de documents scientifiques de niveau recherche, publiés ou non, émanant des établissements d'enseignement et de recherche français ou étrangers, des laboratoires publics ou privés. 


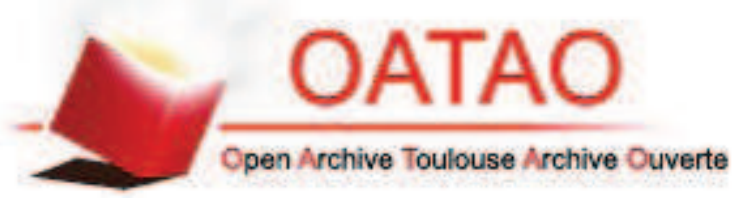

Open Archive TOULOUSE Archive Ouverte (OATAO)

OATAO is an open access repository that collects the work of Toulouse researchers and makes it freely available over the web where possible.

This is an author-deposited version published in : http://oatao.univ-toulouse.fr/ Eprints ID : 10247

To link to this article : DOI:10.1017/jfm.2011.419

URL : http://dx.doi.org/10.1017/jfm.2011.419

To cite this version : Assemat, Pauline and Fabre, David and Magnaudet, Jacques The onset of unsteadiness of two-dimensional bodies falling or rising freely in a viscous fluid: a linear study. (2012) Journal of Fluid Mechanics, vol. 690 . pp. 173-202. ISSN 0022-1120

Any correspondance concerning this service should be sent to the repository administrator: staff-oatao@ listes-diff.inp-toulouse.fr 


\title{
The onset of unsteadiness of two-dimensional bodies falling or rising freely in a viscous fluid: a linear study
}

\author{
Pauline Assemat $^{1}$, David Fabre ${ }^{1}$ and Jacques Magnaudet ${ }^{1,2} \dagger$ \\ ${ }^{1}$ INPT, UPS, IMFT (Institut de Mécanique des Fluides de Toulouse), Université de Toulouse, \\ Allée Camille Soula, 31400 Toulouse, France \\ 2 CNRS, IMFT, 31400 Toulouse, France
}

We consider the transition between the steady vertical path and the oscillatory path of two-dimensional bodies moving under the effect of buoyancy in a viscous fluid. Linearization of the Navier-Stokes equations governing the flow past the body and of Newton's equations governing the body dynamics leads to an eigenvalue problem, which is solved numerically. Three different body geometries are then examined in detail, namely a quasi-infinitely thin plate, a plate of rectangular cross-section with an aspect ratio of 8 , and a rod with a square cross-section. Two kinds of eigenmodes are observed in the limit of large body-to-fluid mass ratios, namely 'fluid' modes identical to those found in the wake of a fixed body, which are responsible for the onset of vortex shedding, and four additional 'aerodynamic' modes associated with much longer time scales, which are also predicted using a quasi-static model introduced in a companion paper. The stability thresholds are computed and the nature of the corresponding eigenmodes is investigated throughout the whole possible range of mass ratios. For thin bodies such as a flat plate, the Reynolds number characterizing the threshold of the first instability and the associated Strouhal number are observed to be comparable with those of the corresponding fixed body. Other modes are found to become unstable at larger Reynolds numbers, and complicated branch crossings leading to mode switching are observed. On the other hand, for bluff bodies such as a square rod, two unstable modes are detected in the range of Reynolds number corresponding to wake destabilization. For large enough mass ratios, the leading mode is similar to the vortex shedding mode past a fixed body, while for smaller mass ratios it is of a different nature, with a Strouhal number about half that of the vortex shedding mode and a stronger coupling with the body dynamics.

Key words: flow-structure interactions, instability, vortex shedding

\section{Introduction}

Understanding and predicting the path of bodies in free fall or rise within a viscous fluid is a problem that is relevant in a broad range of applications (e.g. meteorology, aerospace and chemical engineering, biology, etc.). This issue is a 
long-standing problem in fluid mechanics and is currently the subject of active research - see Ern et al. (2012) and references therein for an up-to-date review. Experimental and computational studies considering either two-dimensional bodies (falling cards, long cylinders) or three-dimensional axisymmetric bodies (spheres, discs, flat cylinders, bubbles, etc.) have revealed a rich variety of dynamical behaviours and complicated bifurcation sequences - see e.g. Jenny, Dusek \& Bouchet (2004) for spheres; Pesavento \& Wang (2004) and Andersen, Pesavento \& Wang (2005a,b) for thin plates; and Auguste (2010) for thick discs. However, the very first transition, which generically leads to a switch from a steady, vertical trajectory to an oscillating path, has received little attention - see, however, Alben (2008) for the case of a rising or flapping ellipse. Our present research effort aims to fill this gap by performing a linear stability analysis of the fully coupled fluid-body system.

In a companion paper (Fabre, Assemat \& Magnaudet 2011, hereafter referred to as FAM), we investigated the particular case of nominally two-dimensional bodies (plates, cylinders, rods) much heavier than the surrounding fluid. In this case, the weak accelerations of the body resulting from its large inertia make it possible to derive rigorously a 'quasi-static' model in which the force and torque are assumed to depend linearly upon the kinematic parameters of the motion (specifically the velocity variation, incidence angle and rotation rate). This model predicts the existence of four modes with slow time scales, which will be referred to as 'aerodynamic' modes. Two of them, called 'back to terminal velocity' (BTV) and 'back to vertical' (BV), are non-oscillating and are always damped (i.e. they are associated with a real, negative eigenvalue). The last two modes are associated with a pair of complex conjugate eigenvalues. They describe a slow, oscillating motion (this is why they are referred to as 'low-frequency' (LF) modes) that, at leading order, can be explained by the existence of a torque proportional to the incidence angle. This torque acts as a restoring force as soon as the body short axis is no longer aligned with its path. In the case of thin plates, this oscillating mode was found to be stable whatever the Reynolds number. In contrast, for bluff bodies like a square rod, this mode was found to become unstable for a critical Reynolds number close to that corresponding to the onset of vortex shedding past the same body held fixed. This finding suggests that a competition between two kinds of oscillating modes with a different nature could occur for such bluff bodies.

The 'quasi-static' approach developed in FAM assumes that the forces acting on the body depend only on its instantaneous position and velocity, and obviously does not allow one to account for corrections due to unsteadiness of the body motion (through the so-called added-mass and history terms), nor for the intrinsic dynamics of the wake (through vortex shedding effects). Therefore, in order to address the general case of bodies whose inertia is comparable to or lower than that of the fluid, we have to call upon a more general approach. The purpose of the present paper is to develop such an approach by applying a global linear stability analysis to the fully coupled fluid-body problem (restricting ourselves, as in FAM, to two-dimensional geometries). As expected from physical intuition, the results will reveal that, the smaller the bodyto-fluid relative density, the larger the differences between the predictions provided by the two approaches (see for instance figure 6).

Besides the fundamental interest of providing a mapping of the primary bifurcation threshold as a function of the geometrical characteristics of the body and of its inertia relative to the fluid, the present approach is expected to improve the understanding of the general problem in at least two ways. First, we expect to gain some insight regarding the physical mechanism responsible for the primary instability, and in 
particular to disentangle the respective effect of the intrinsic wake dynamics (vortex shedding) and of the aerodynamic efforts experienced by the body. Second, the present approach allows us to identify the dominant modes of the problem. Hence it constitutes the first step towards a rational modelling of the nonlinear dynamics of the body plus fluid system using bifurcation theory, an approach that has proved successful for the related, although simpler, problem of the wake of fixed axisymmetric bodies (Fabre, Auguste \& Magnaudet 2008; Auguste, Fabre \& Magnaudet 2009; Meliga, Chomaz \& Sipp 2009).

The paper is organized as follows. In $\S 2$ we set up the description and parameterization of the physical problem, establish the system of governing equations and introduce the linear stability approach and the coupled linear system to be solved. The numerical method employed to solve the eigenvalue problem and some validations are presented in $\S 3$. Results for a quasi-infinitely thin plate $(\S 4)$, and for two other geometries of interest, namely a rectangular rod with an aspect ratio of $8(\S 5)$ and a square rod ( $\$ 6)$, are then discussed. Section 7 summarizes the main findings of the present investigation and opens up some perspectives.

\section{Statement of the problem}

\subsection{Geometry and dimensionless parameters}

We consider bodies with a uniform cross-section in the $(\boldsymbol{x}, \boldsymbol{y})$ plane that are sufficiently elongated in the $z$ direction to allow a two-dimensional treatment. In what follows, $\ell$ is the characteristic width of the body, $d$ its thickness, $A$ its cross-section (with $A=\ell d$ for a rectangular plate) and $m$ its mass per unit length; $g$ denotes gravity while $\rho$ and $v$ are the uniform density and viscosity of the fluid, respectively. These quantities allow the problem to be entirely characterized by three dimensionless parameters, namely

$$
m^{*}=m /\left(\rho \ell^{2}\right), \quad \chi=\ell / d, \quad A r=\frac{1}{v} \sqrt{|m-\rho A| \frac{g \ell}{\rho}} \equiv \frac{1}{v} \sqrt{\left|m^{*}-\frac{A}{\ell^{2}}\right| g \ell^{3}} .
$$

The parameter $m^{*}$ characterizes the body-to-fluid mass ratio, while $\chi$ is the body geometrical aspect ratio. The parameter $A r$ is the so-called Archimedes number, which compares gravitational and viscous effects. This parameter can thus be thought of as a Reynolds number based on the body length scale $\ell$ and the gravitational velocity scale defined as $U_{g}=\sqrt{|m-\rho A| g /(\rho \ell)}$, which differs from the actual falling/rising speed (indeed, as shown by (2.13) below, $U_{g}$ coincides with the falling/rising speed $U_{0}$ only if the drag coefficient equals 2 ). The Archimedes number is frequently called 'Reynolds number' in available studies dealing with freely moving bodies. To avoid any confusion, we prefer to keep the terminology 'Reynolds number' for the parameter built on the actual falling velocity of the body. Note that, since this velocity is not imposed externally, the Reynolds number is not a primary control parameter from an experimental or computational point of view.

Alternative choices for normalizing the body mass could have been selected. The most obvious one would be the density ratio $\rho_{s} / \rho$ between the solid and fluid. However, this ratio becomes infinite in the case of infinitely thin plates, making it inappropriate for the present study (one has $\rho_{s} / \rho=\chi m^{*}$ for rectangles of finite width). Another possibility frequently used in studies of falling cards involves the moment of inertia $\mathscr{I}$ and defines the dimensionless quantity $\mathscr{I}^{*}=32 \mathscr{I} / \pi \rho \ell^{4}$ (Smith 1971; Andersen et al. 2005b), which is related to $m^{*}$ through $\mathscr{I}^{*}=32 / \pi\left[\mathscr{I} / m \ell^{2}\right] m^{*}$, where 


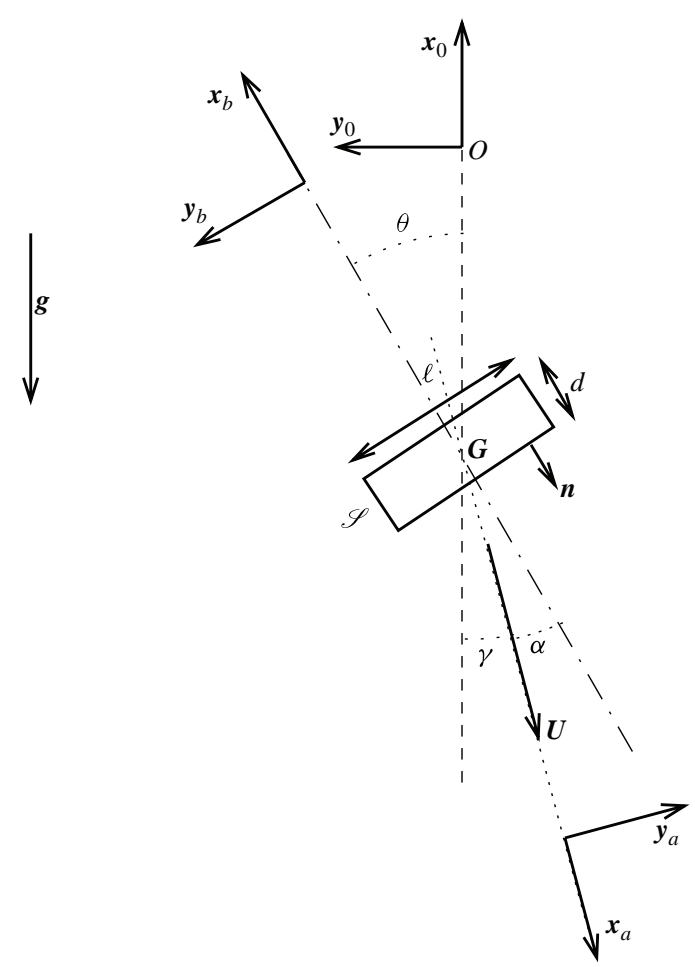

FIGURE 1. Sketch of the problem defining the three reference frames and the angles used throughout the paper.

the ratio $\left[\mathscr{I} / m \ell^{2}\right]$ is a constant for a given geometry (one has $\mathscr{I}^{*}=8\left(1+1 / \chi^{2}\right) m^{*} / 3 \pi$ for a rectangular plate).

Figure 1 illustrates the generic geometry of the problem and specifies the notation used throughout the paper. The body is bounded by a contour $\mathscr{S}$ whose outgoing unit normal is $\boldsymbol{n}$. For future purposes, we define three distinct reference frames. The frame $\left(O, \boldsymbol{x}_{0}, \boldsymbol{y}_{0}, \boldsymbol{z}\right)$ is fixed with respect to the laboratory and assumed to be inertial, the unit vector $\boldsymbol{x}_{0}$ and the gravity vector $\boldsymbol{g}$ being parallel or antiparallel, depending on whether the body rises or falls through the fluid. Hence the natural direction of motion is $-\boldsymbol{x}_{0}$ in both cases. We then define the reference frame $\left(G, \boldsymbol{x}_{b}, \boldsymbol{y}_{b}, \boldsymbol{z}\right)$ attached to the body ( $G$ is the body centre of mass), and assume that the unit vector $\boldsymbol{y}_{b}$ is always parallel to the long axis of the body cross-section. Finally, we introduce the aerodynamic (or Frenet) reference frame $\left(G, \boldsymbol{x}_{a}, \boldsymbol{y}_{a}, \boldsymbol{z}\right)$, in which the unit vector $\boldsymbol{x}_{a}$ is collinear to the body velocity $\boldsymbol{U}$, i.e. $\boldsymbol{U}=\boldsymbol{U} \boldsymbol{x}_{a}$. The angles $\theta$ and $\gamma$ defined in figure 1 correspond to the inclination of the body and of the path with respect to the vertical, respectively. The difference $\alpha=\theta-\gamma$ thus characterizes the drift between the body and its path, i.e. $\alpha$ may be thought of as the incidence angle. Note, however, that, in the base state, buoyancy-driven bodies have their broad side horizontal, so that $\alpha=0$ corresponds to a $\pi / 2$ angle between the body velocity and its largest dimension, which contrasts with the 'aerodynamic' definition according to which the incidence angle is zero when the body velocity is parallel to the chord. In the body reference frame, the velocity can be written as $\boldsymbol{U}=U_{x} \boldsymbol{x}_{b}+U_{y} \boldsymbol{y}_{b}$, with $U_{x}=U \cos \alpha$ and $U_{y}=U \sin \alpha$. Finally, the body rotation rate is $\Omega=\Omega z$ with $\Omega=\mathrm{d} \theta / \mathrm{d} t$. 


\subsection{Equations of motion}

In the body reference frame, the Navier-Stokes equations governing the incompressible flow past the body and Newton's laws governing the body motion can be written as

$$
\begin{gathered}
\boldsymbol{\nabla} \cdot \boldsymbol{V}=0 \\
\partial_{t} \boldsymbol{V}+\boldsymbol{V} \cdot \nabla \boldsymbol{V}=-\frac{1}{\rho} \nabla P+v \nabla^{2} \boldsymbol{V} \\
-\left(\frac{\mathrm{d} \boldsymbol{U}}{\mathrm{d} t}+\boldsymbol{\Omega} \wedge \boldsymbol{U}+\frac{\mathrm{d} \boldsymbol{\Omega}}{\mathrm{d} t} \wedge \boldsymbol{r}+\boldsymbol{\Omega} \wedge(\boldsymbol{\Omega} \wedge \boldsymbol{r})+2 \boldsymbol{\Omega} \wedge \boldsymbol{V}\right), \\
m \frac{\mathrm{d} \boldsymbol{U}}{\mathrm{d} t}+m \boldsymbol{\Omega} \wedge \boldsymbol{U}=(m-\rho A) \boldsymbol{g}+\int_{\mathscr{S}} \boldsymbol{T} \cdot \boldsymbol{n} \mathrm{d} l, \\
\mathscr{I} \frac{\mathrm{d} \boldsymbol{\Omega}}{\mathrm{d} t}=\int_{\mathscr{S}} \boldsymbol{r} \wedge(\boldsymbol{T} \cdot \boldsymbol{n}) \mathrm{d} l,
\end{gathered}
$$

where $\boldsymbol{V}$ is the relative fluid velocity, $P$ the pressure, $v$ the kinematic viscosity, $\boldsymbol{r}$ the distance with respect to $G$ and $\boldsymbol{T}=-P \boldsymbol{I}+\rho \nu\left(\nabla \boldsymbol{V}+{ }^{\mathrm{t}} \nabla \boldsymbol{V}\right)$ the stress tensor, $\boldsymbol{I}$ denoting the Kronecker tensor.

Since the no-slip boundary condition is assumed to hold at the body surface and the fluid is at rest at infinity, one has

$$
\boldsymbol{V}=\mathbf{0} \quad \text { on } \mathscr{S} \quad \text { and } \quad \boldsymbol{V}=-(\boldsymbol{U}+\boldsymbol{\Omega} \wedge \boldsymbol{r}) \text { for }\|\boldsymbol{r}\| \rightarrow \infty .
$$

Since the problem considered here is two-dimensional, the whole solution can be represented by the state vector $\mathscr{X}$ such that

$$
\mathscr{X}=[\boldsymbol{V}(x, y, t), P(x, y, t), \boldsymbol{U}(t), \Omega(t), \theta(t)],
$$

with $\Omega(t)=\Omega(t) \cdot z$. The first two components of $\mathscr{X}$ are a two-dimensional vector field (the fluid velocity) and a scalar field (the pressure) describing the flow around the body, respectively, while the latter three components are a two-dimensional vector (the body velocity) and two scalars (the rotation rate and inclination angle) describing the body kinematics, respectively.

\subsection{Perturbation method}

To perform a linear stability analysis, the state vector is expanded in the form

$$
\mathscr{X}=\mathscr{X}_{0}+\mathscr{X}_{1} \mathrm{e}^{\lambda t} .
$$

Here $\mathscr{X}_{0}=\left[\boldsymbol{V}_{0}(x, y), P_{0}(x, y),-U_{0} \boldsymbol{x}_{b}, 0,0\right]$ corresponds to the base state in which the wake is steady and symmetric and $\mathscr{X}_{1}=[\hat{\boldsymbol{v}}(x, y), \hat{p}(x, y), \hat{\boldsymbol{u}}, \hat{\omega}, \hat{\theta}]$ is the perturbation (the ^symbol is used to indicate that the components of the state vector are complex). The perturbation is sought in the form of an eigenmode with an associated eigenvalue $\lambda=\lambda^{r}+\mathrm{i} \lambda^{i}$, where $\lambda^{r}$ is the growth rate and $\lambda^{i}$ the oscillation frequency. In what follows the latter will frequently be normalized so as to define the so-called Strouhal number $S t=\lambda^{i} \ell / 2 \pi U_{0}$. Following the usual convention, only the real part of complex quantities is to be retained in (2.8) and in subsequent equations.

\subsubsection{Order 0: base flow}

The leading-order state vector $\left[\boldsymbol{V}_{0}(x, y), P_{0}(x, y),-U_{0} \boldsymbol{x}_{b}, 0,0\right]$ corresponds to the steady vertical fall/rise of the body, the body short axis being aligned with its path (hence $\boldsymbol{x}_{b}=\boldsymbol{x}_{0}$ ). The associated continuity and momentum equations read

$$
\nabla \cdot V_{0}=0,
$$




$$
\mathbf{0}=-\boldsymbol{V}_{0} \cdot \nabla \boldsymbol{V}_{0}-\frac{1}{\rho} \nabla P_{0}+v \nabla^{2} \boldsymbol{V}_{0},
$$

while the corresponding boundary conditions are

$$
\boldsymbol{V}=\mathbf{0} \quad \text { on } \mathscr{S} \text { and } \quad \boldsymbol{V}_{0}=-U_{0} \boldsymbol{x}_{b} \text { for }\|\boldsymbol{r}\| \rightarrow \infty,
$$

$U_{0}$ being the rise/fall velocity directed along $\boldsymbol{x}_{b}$. Equations (2.9)-(2.11) are formally similar to those governing the flow about the same body forced to move at a constant speed $U_{0} \boldsymbol{x}_{b}$, or equivalently about the same body held fixed within a uniform incident stream $-U_{0} \boldsymbol{x}_{b}$. The vertical projection of Newton's equation reads

$$
(m-\rho A) \boldsymbol{g}=-\int_{\mathscr{S}} \boldsymbol{T}_{0} \cdot \boldsymbol{n} \mathrm{d} l \equiv-D_{0} \boldsymbol{x}_{0},
$$

where $D_{0}$ is the drag and $\boldsymbol{T}_{0}$ denotes the base-state stress tensor. Since the buoyancycorrected weight changes sign for $m=\rho A$, rectangular bodies fall if $m^{*}>1 / \chi$ and rise if $0<m^{*}<1 / \chi$ (this implies that, for a given $m^{*}>0$, infinitely thin plates with $\chi \rightarrow \infty$ always fall). Following usual aerodynamic conventions, the drag may be written as $D_{0}=\rho \ell U_{0}^{2} C_{d}(R e) / 2$, where $R e=U_{0} \ell / \nu$ is the nominal Reynolds number. With these definitions, (2.12) takes the form

$$
A r^{2}=\frac{R e^{2} C_{d}(R e)}{2} .
$$

In what follows the results will be presented using the above Reynolds number. Indeed, when solving the base-flow (2.9)-(2.11) it is natural to normalize velocities and lengths with $U_{0}$ and $\ell$, respectively. This choice also simplifies comparisons with the corresponding fixed-body problem. In experiments, a mean Reynolds number based on the average velocity of the body is often used. Whenever the rectilinear path is stable, the drag experienced by the body equals $D_{0}$ and the mean Reynolds number equals the nominal Reynolds number $R e$. However, when the rectilinear path is unstable, the unsteady dynamics in the wake generally provide an additional drag, so that the mean Reynolds number is somewhat lower than Re. In such circumstances, comparisons with the present theory should rather be based on the Archimedes number.

\subsubsection{Order 1: unsteady modes}

Injecting the expansion (2.8) into the governing equations and retaining terms proportional to $\mathscr{X}_{1}$ yields

$$
\begin{gathered}
\nabla \cdot \hat{\boldsymbol{v}}=0, \\
\lambda(\hat{\boldsymbol{v}}+\hat{\boldsymbol{u}}+\hat{\omega} \boldsymbol{z} \wedge \boldsymbol{r})=-\hat{\boldsymbol{v}} \cdot \nabla \boldsymbol{V}_{0}-\boldsymbol{V}_{0} \cdot \nabla \hat{\boldsymbol{v}}-\frac{1}{\rho} \nabla \hat{p}+v \nabla^{2} \hat{\boldsymbol{v}} \\
-2 \hat{\omega} z \wedge \boldsymbol{V}_{0}-\hat{\omega} \boldsymbol{z} \wedge \boldsymbol{U}_{0}, \\
m \lambda \hat{\boldsymbol{u}}=-m \hat{\omega} \boldsymbol{z} \wedge \boldsymbol{U}_{0}+(m-\rho A) g \hat{\theta} \boldsymbol{y}_{b}+\int_{\mathscr{S}} \hat{\boldsymbol{T}} \cdot \boldsymbol{n} \mathrm{d} l, \\
\mathscr{I} \lambda \hat{\omega}=\int_{\mathscr{S}} \boldsymbol{r} \wedge(\hat{\boldsymbol{T}} \cdot \boldsymbol{n}) \mathrm{d} l, \\
\lambda \hat{\theta}=\hat{\omega},
\end{gathered}
$$


where $\hat{\boldsymbol{T}}$ denotes the first-order disturbance to the stress tensor. The associated boundary conditions are

$$
\hat{\boldsymbol{v}}=\mathbf{0} \quad \text { on } \mathscr{S} \quad \text { and } \quad \hat{\boldsymbol{v}}=-(\hat{\boldsymbol{u}}+\hat{\omega} z \wedge \boldsymbol{r}) \quad \text { for }\|\boldsymbol{r}\| \rightarrow \infty .
$$

Together, these equations lead to a generalized eigenvalue problem of the form $\mathscr{A} \mathscr{X}_{1}=\lambda \mathscr{B} \mathscr{X}_{1}$, where $\mathscr{A}$ and $\mathscr{B}$ are linear non-symmetric operators. As is well known, this problem has non-trivial solutions only for values $\lambda$ whose set constitutes the eigenvalue spectrum of the problem. The variables involved in the eigenvalue problem have different physical dimensions. To make them dimensionless, all computations are carried out using $d=1, U_{0}=1, \rho=1$.

As the base flow is symmetric, the eigenmodes can be split into two distinct sets of symmetric and antisymmetric modes, respectively. The numerical solution procedure described in the next section takes advantage of this property. Symmetric modes are only coupled to the axial motion, i.e. they are such that $\hat{\boldsymbol{u}}=\hat{u}_{x} \boldsymbol{x}_{b}, \hat{\omega}=0$, $\hat{\theta}=0$. Consequently these modes can only result in a drag on the body. In contrast, antisymmetric modes can exert a lift and a torque but no drag, and are thus coupled to the inclination, rotation and lateral body velocity, i.e. they are such that $\hat{\boldsymbol{u}}=\hat{u}_{y} \boldsymbol{y}_{b}$. When discussing the structure of these modes, it will be found useful to introduce their incidence angle component $\hat{\alpha}$, defined as the angle between the actual body velocity $-U_{0} \boldsymbol{x}_{b}+\hat{\boldsymbol{u}}$ and the vector $\boldsymbol{x}_{b}$ attached to the body (see figure 1). Comparing expressions of the body velocity in the relative and aerodynamic reference frames (i.e. $U \boldsymbol{x}_{a} \approx-U_{0} \boldsymbol{x}_{b}+\hat{u}_{y} \boldsymbol{y}_{b}$ ) indicates that the incidence angle of a given eigenmode is directly related to the corresponding lateral velocity component through $\hat{\alpha}=\hat{u}_{y} / U_{0}$.

As usual, the eigenvectors resulting from the eigenvalue computation are defined up to an arbitrary amplitude and a normalization condition has to be imposed to compare the effects of the various modes. For reasons that will be made clear later, antisymmetric modes are normalized in such a way that the inclination of the body is

unity, i.e. $\hat{\theta}=1$, while the normalization of symmetric modes is chosen such that the corresponding velocity increment is unity, i.e. $\hat{u}_{x}=1$.

\section{Numerical method and validation}

\subsection{Finite element method}

The computations performed in the present study were carried out using the finite element software FreeFEM++ (see http://www.freefem.org). This computational approach was originally introduced in the context of hydrodynamic instabilities by Sipp \& Lebedev (2007). It may be summarized as follows. First, the base flow is calculated via a Newton method, whose advantage is to be capable of capturing both stable and unstable steady-state solutions. The associated Jacobian matrix is inverted using the UMFPACK library. Then the eigenvalue problem is solved via a Krylov-Shur technique implemented in the SLEPc library (see http://www.grycap. upv.es/slepc/). Thanks to the capabilities offered by this library, the non-symmetric generalized eigenvalue problem may be solved on a parallel computer.

Figure 2 shows the computational domain and the associated numerical parameters. The domain size is characterized by lengths $\left(l_{1}, l_{2}, h_{1}\right)$ indicated on the figure. An unstructured grid based on triangular cells is generated using the built-in grid generator of the software FreeFEM++. The grid includes local refinement along the body surface, with extra refinement at the corners. Additional fictitious inner boundaries (displayed with dashed lines in the figure) are used to constrain the grid density in the wake. Taking advantage of the aforementioned symmetries, computations of the base flow 


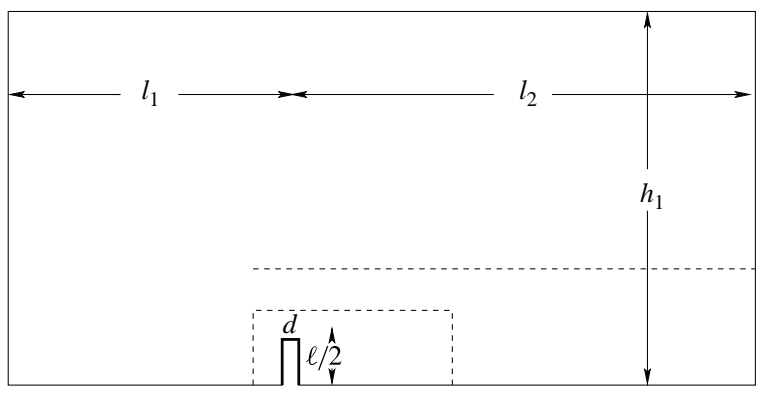

FIGURE 2. Sketch of the computational domain. Thick solid lines correspond to the body surface, thin solid lines to the outer boundary of the domain and dashed lines to fictitious inner boundaries used to refine the grid in the wake region.

are performed within a half-domain by imposing suitable symmetry conditions along the symmetry plane. The stability equations are also solved within a half-domain by taking advantage of the splitting into symmetric and antisymmetric eigenmodes, each family having its own set of symmetry conditions. The fields $[\boldsymbol{V}, P]$ are approximated by quadratic and linear elements using a weak formulation, respectively.

For both the base flow and the eigenvalue computations, the condition at infinity is imposed at the inlet $\left(x=-l_{1}\right)$ and on the lateral boundary $\left(y=h_{1}\right)$, while a weaker condition corresponding to the vanishing of the tangential stress is imposed at the outlet $\left(x=l_{2}\right)$.

\subsection{Grid convergence}

In this section we show sample results obtained with five grids labelled $0-4$. The configuration chosen in these tests corresponds to a thin plate at zero incidence with an aspect ratio $\chi=10^{4}, \operatorname{Re}=30$ and $m^{*}=5$. The value $\chi=10^{4}$ is actually chosen as an approximation of the ideal case of an infinitely thin plate $(\chi \rightarrow \infty)$, as our numerical approach requires the plate to have a finite thickness. However, we checked that the results are insensitive to the actual value of $\chi$ provided it is sufficiently large, as well as to the discretization along the plate edge: there is only one grid point along the edge in the computations whose results are discussed below but a better resolution along the edge leaves all results unchanged. Table 1 provides the dimensions $\left(l_{1}, l_{2}, h_{1}\right)$ of the computational domain (see figure 2), the grid density measured by the number of degrees of freedom (d.o.f.) in the variational formulation, and the computed values of three eigenvalues. Eigenvalues $\lambda_{B_{1}}$ and $\lambda_{B_{2}}$ correspond to the two least damped antisymmetric modes, while $\lambda_{B T V}$ is associated with the least damped symmetric mode. Figure 3 displays the full spectra in the complex $\lambda$ plane computed using those grids. These spectra consist of a small number (five in the present case) of physical eigenvalues located in the right half of the plane plus a large number of damped modes of spurious nature that depend on the detail of the discretization. It is important to notice that, although most damped modes found for a given value of $m^{*}$ and $R e$ are unphysical, it would be incorrect to consider only the least damped of them. Indeed, several unstable branches may cross when $m^{*}$ or $R e$ is varied, as will be seen later (e.g. figures 9 and 10).

Grids 0-2 (figure $3 a$ ) illustrate the influence of node density. The difference between the computed eigenvalues is at most $1.1 \%$ for the physically relevant eigenvalues reported in table 1 . As can be seen in figure 3(a), a number of spurious eigenmodes among the least damped ones are also insensitive to the node density, while the most 

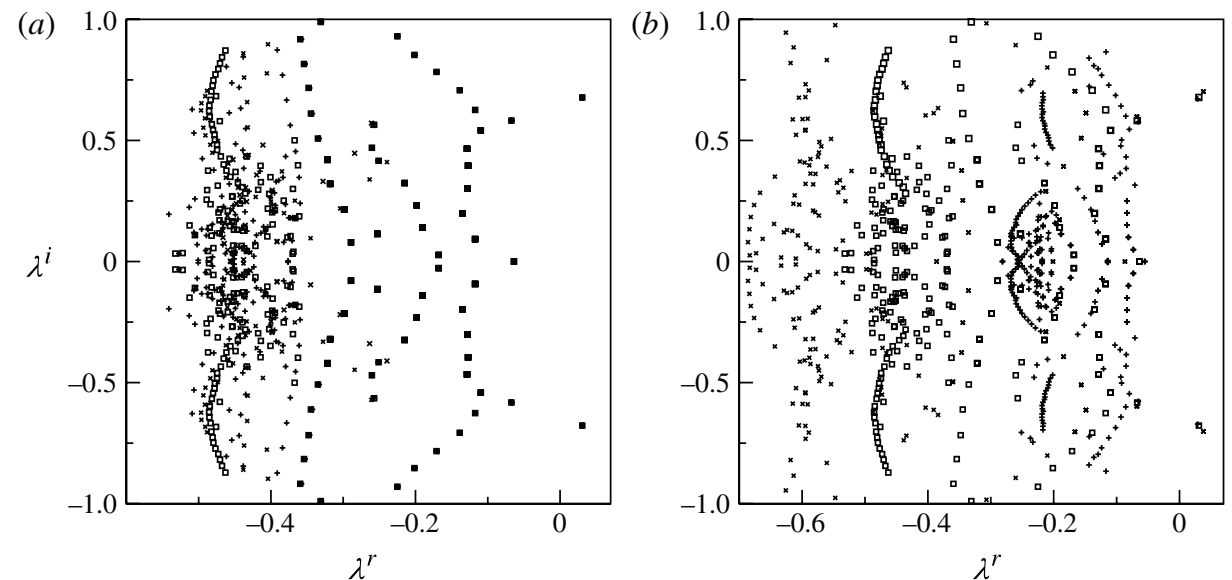

FIGURE 3. Spectra in the complex $\lambda$ plane for a flat plate at zero incidence with $m^{*}=5$ and $R e=30$ : (a) grid $0(\times), 1(\square), 2(+) ;(b)$ grid $1(\square), 3(\times), 4(+)$.

$\begin{array}{lccccccccc}\begin{array}{l}\text { Grid } \\ \text { number }\end{array} & l_{1} & l_{2} & h_{1} & \text { d.o.f. } & \lambda_{B_{1}}{ }^{r} & \lambda_{B_{1}}{ }^{i} & \lambda_{B_{2}}{ }^{r} & \lambda_{B_{2}}{ }^{i} & \lambda_{B T V} \\ 0 & & & & & & & & & \\ 1 & 20 & 40 & 20 & 33469 & 0.03075 & 0.6758 & -0.06783 & 0.5790 & -0.06373 \\ 2 & 20 & 40 & 20 & 80854 & 0.03081 & 0.6774 & -0.06724 & 0.5821 & -0.06376 \\ 3 & 20 & 40 & 20 & 192418 & 0.03078 & 0.6778 & -0.06706 & 0.5827 & -0.06376 \\ 4 & 10 & 35 & 10 & 56009 & 0.03803 & 0.7013 & -0.06775 & 0.5982 & -0.08675 \\ & 30 & 90 & 30 & 172524 & 0.02890 & 0.6716 & -0.06550 & 0.5770 & -0.05516\end{array}$

TABLE 1. Details of the grid convergence study for a flat plate at zero incidence with $m^{*}=5, R e=30$. Dimensions of the computational domain (lengths $l_{1}, l_{2}, h_{1}$ are defined in figure 2), number of degrees of freedom (d.o.f.) and computed values of three eigenvalues $\lambda_{B_{1}}, \lambda_{B_{2}}$ and $\lambda_{B T V}$ for five grids numbered 0-4 (superscripts $r$ and $i$ correspond to the real and imaginary parts of the complex eigenvalues, respectively). Grid 1 is the one with which results discussed in $\S 4$ were obtained. Grids $0-2$ show the influence of the density of nodes, whereas grids 3 and 4 reveal the influence of the size of the domain.

damped modes located in the left part of the figure are clearly grid-dependent. Grids 1,3 and 4 (figure $3 b$ ) illustrate the influence of the dimensions of the computational domain. As can be seen in the figure, grid 3, which has the smallest size, leads to significant deviations in the eigenvalues compared to the other grids, revealing a clear confinement effect. This is an indication that, provided grid density is sufficient, the size of the domain is the most influential parameter. Results obtained with the other two grids (1 and 4) show better agreement. However, the spurious eigenvalues obtained with grid 4 (which has the largest size) are less damped and tend to approach the real axis, with the possible effect of masking the physically meaningful eigenvalues if they come too close to one another. Hence, although the values of the physically meaningful eigenvalues indeed converge when the size of the computational domain is increased, it becomes hard to disentangle them from purely numerical eigenvalues when the domain becomes very large. This issue could be fixed by using a continuation technique to track precisely the variations of the eigenvalues with the domain size, but, given the weak variations of the physically meaningful eigenvalues 

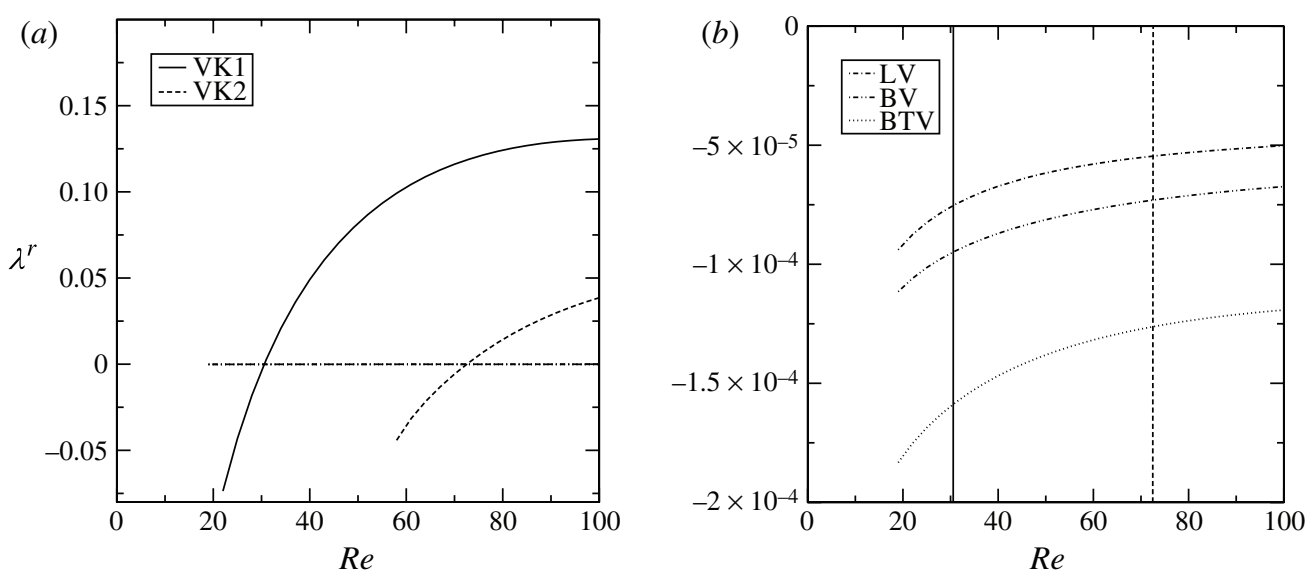

FIGURE 4. Amplification rates $\lambda^{r}$ as a function of the Reynolds number for a heavy flat plate $\left(m^{*}=10^{4}\right)$ : $(a)$ 'fluid' modes VK1 and VK2; $(b)$ zoom for small negative values of $\lambda^{r}$ revealing the 'aerodynamic' modes BTV, BV and LF.

$\begin{array}{llllllllll}m^{*} & R e & \lambda_{V K 1}{ }^{r} & \lambda_{V K 1}{ }^{i} & \lambda_{V K 2}{ }^{r} & \lambda_{V K 2}{ }^{i} & \lambda_{B T V} & \lambda_{B V} & \lambda_{L F}{ }^{r} & \lambda_{L F}{ }^{i} \\ 10000 & 30 & -0.00375 & 0.6505 & - & - & -0.00016 & -0.000095 & -0.000076 & 0.0141 \\ & 73 & 0.11894 & 0.5036 & 0.00098 & 0.5568 & -0.00013 & -0.000073 & -0.000054 & 0.0115\end{array}$

TABLE 2. Selection of computed eigenvalues for a flat plate with $m^{*}=10^{4}$. Note that the VK2 eigenvalue could not be obtained for $R e=30$, owing to its proximity to spurious eigenmodes.

between grids 1 to 2 , we found this unnecessary. Therefore, in the present study the optimal choice of the domain size results from a compromise between a large enough size required to get rid of confinement effects and a small enough size to avoid contamination of the physical meaningful modes by the spurious modes. The results reported below with a flat plate were obtained with grid 1, which provides a good compromise between accuracy, spectrum spreading and computational time.

\section{The thin plate at zero incidence}

In this section we focus on the case of a thin plate with a rectangular cross-section of nearly infinite aspect ratio (actually $\chi=10^{4}$ ). We first present results obtained in the limit of large mass ratios $\left(m^{*} \gg 1\right)$, a regime where a rational classification of the eigenmodes is possible. This also allows us to introduce a number of criteria that can be used to analyse the structure of the eigenmodes. Then we discuss the results of the parametric study performed within the $\left(m^{*}, R e\right)$ plane and describe the structure of the eigenmodes responsible for the destabilization of the physical system.

\subsection{Very heavy plates $\left(m^{*} \gg 1\right)$}

We first consider the case of a very heavy plate with $m^{*}=10^{4}$. Figure $4(a)$ shows the real part of the computed eigenvalues in the range $\operatorname{Re} \in[0,100]$. The figure clearly reveals two sets of eigenvalues with highly contrasted magnitudes. 


\subsubsection{The 'fluid' modes VK1 and VK2}

The eigenmodes associated with the first two unstable branches are both antisymmetric. We call them 'fluid' modes, as the corresponding eigenvalues are very close to those found in the case where the plate is held fixed. Indeed, our calculations concerning the stability of a fixed plate (not shown) also reveal two eigenmodes, which become successively unstable as the Reynolds number is increased. The corresponding thresholds and Strouhal numbers are $R e_{c 1}=30.57, S t_{c 1}=0.103$ and $R e_{c 2}=72.55, S t_{c 2}=0.089$. According to figure 4, results obtained for $m^{*}=10^{4}$ reveal very close characteristics, namely $R e_{V K 1}=30.56, S t_{V K 1}=0.103$ and $R e_{V K 2}=$ 72.54, $S t_{V K 2}=0.089$, making the one-to-one connection with the fixed-plate modes obvious. We call the first mode 'VK1' as it is associated with the onset of vortex shedding leading to the celebrated Von Kármán street. The corresponding threshold $R e_{V K 1}$ lies in the range of the results obtained by Saha (2007), who found $30<R e_{c 1}<35$ for a fixed plate. Jackson (1987) reported a significantly lower threshold, namely $R e_{c 1}=27.77$, and a higher Strouhal number, $S t_{c 1}=0.123$, but the size of the computational domain he used corresponds to $l_{1}=5, l_{2}=15$ and $h_{1}=5$ in the terminology of $\S 3$. Hence the corresponding flow is expected to be strongly constrained by the outer boundary and the difference with present predictions is consistent with those observed between grids 0 and 3 in the previous section.

The second 'fluid' mode, denoted as 'VK2', is associated with a lower Strouhal number and a much higher threshold. To the best of our knowledge, the existence of such a secondary mode has not been reported in the literature. This mode is not seen in direct numerical simulations (the second transition occurring in the flow past a fixed plate leads to a three-dimensional wake structure for $105<R e<110$; see Thompson et al. 2006). However, this VK2 mode is a solution of the eigenvalue problem and may play a role in the nonlinear interaction process between modes.

Figure 5 illustrates the structure of the base flow and of the two 'fluid' modes VK1 and VK2 for $m^{*}=10^{4}$. The vorticity is depicted together with some streamlines of the base flow, which allow the visualization of the recirculation zone. When representing unsteady modes such as those of figure $5(b, c)$, we adopt the convention that the left half of the figure displays the real part of the mode whereas the right half displays its imaginary part. These representations will be used throughout the paper and the corresponding zooms will always focus on the subdomain $[-1,21] \times[-5,5]$ in the $\left(\boldsymbol{x}_{b}, \boldsymbol{y}_{b}\right)$ axes. As revealed by the figure, the VK1 mode is characterized by alternating vortical structures of opposite sign, which are convected downstream in the wake, in agreement with known results (see e.g. Natarajan \& Acrivos 1993). The VK2 mode has a similar structure but its amplitude increases downstream with the distance to the body.

To interpret the very large vorticity maxima seen in figure $5(b, c)$, one has to keep in mind that each eigenmode has been normalized by setting the inclination angle $\hat{\theta}$ to unity. Thus what these large maxima mean is that the vortical component of the flow must be extremely strong in order for the inclination of the plate to reach an $O(1)$ value. In other words, the lateral motion and angular deviation of the plate associated with these modes are much weaker than the vortical fluid motion. This is in line with the intuitive idea that, for heavy bodies, the occurrence of vortex shedding has almost no effect on the path.

\subsubsection{The 'aerodynamic' BTV, BV and LF modes}

In addition to the two 'fluid' modes, figure $4(b)$ reveals the existence of four additional eigenvalues (two are real and the other two are complex conjugates) with 

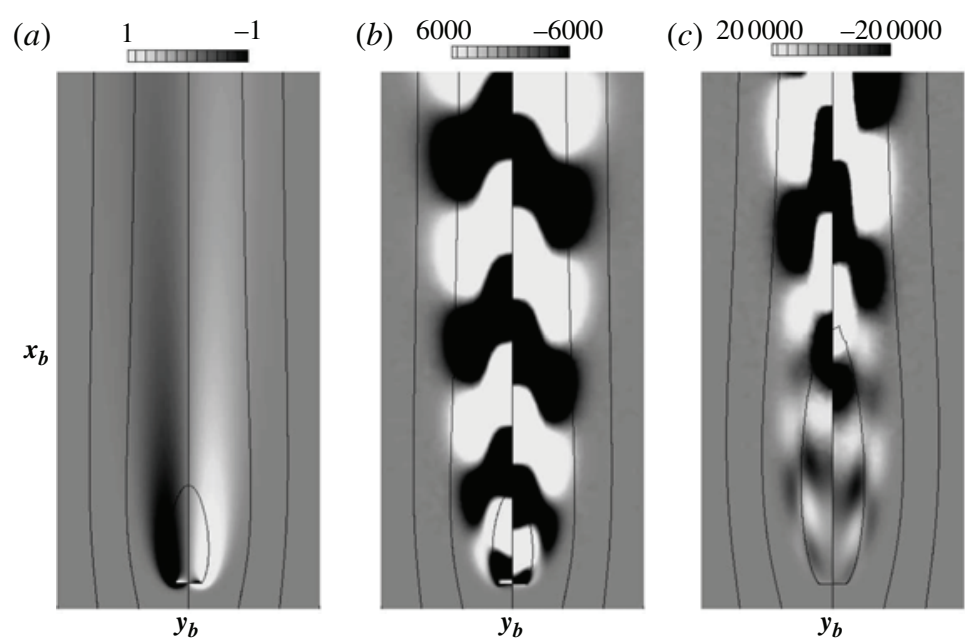

FIgURE 5. Structure of the base flow and of the 'fluid' eigenmodes of a flat plate with $m^{*}=10^{4}$ : (a) base state for $R e=30 ;(b) \mathrm{VK} 1$ mode for $R e=30$; and $(c) \mathrm{VK} 2$ mode for $R e=73$. The greyscale follows the iso-levels of the vorticity, dark (respectively, light) zones corresponding to negative (respectively, positive) values. The left (respectively, right) side of the last two panels corresponds to the real (respectively, imaginary) part of the eigenmode. In all cases the solid lines correspond to streamlines of the base flow at the same Reynolds number (note the closed streamlines at the back of the plate).

a much smaller magnitude. These particular modes were investigated in a companion paper (FAM) using a quasi-static approach. This approach relies on the assumption that the forces and torque on the body depend linearly upon the incremental streamwise velocity $u=\boldsymbol{u} \cdot \boldsymbol{x}_{b}$, the incidence angle $\alpha$ and the rotation rate $\omega$ in the form

$$
D=D_{0}+D_{, u} u, \quad L=L_{, \alpha} \alpha+L_{, \omega} \omega, \quad M=M_{, \alpha} \alpha+M_{, \omega} \omega .
$$

In FAM, the coefficients $D_{, u}, L_{, \alpha}, L_{, \omega}, M_{, \alpha}$ and $M_{, \omega}$ entering the model were determined by solving three elementary problems in which the base flow is respectively perturbed by a constant incremental velocity, a constant incidence and a constant rotation rate. Introducing these coefficients in the equations governing the body motion in the aerodynamic reference frame yields a coupled system of the form

$$
\begin{gathered}
m \dot{u}=-D_{, u} u, \\
m U_{0} \dot{\gamma}=-D_{0} \gamma-L_{, \alpha} \alpha-L_{, \omega} \omega, \\
I \dot{\omega}=M_{, \alpha} \alpha+M_{, \omega} \omega, \\
\omega=\dot{\alpha}+\dot{\gamma} \quad(=\dot{\theta}) .
\end{gathered}
$$

In the limit of large mass ratios, the eigenvalues can be approached using a regular asymptotic expansion, which yields in dimensional form

$$
\begin{gathered}
\lambda_{B T V} \approx-\frac{1}{m} D_{, u}, \\
\lambda_{B V} \approx-\frac{D_{0}}{m U_{0}}, \quad \lambda_{L F} \approx \pm i \sqrt{\frac{\left|M_{, \alpha}\right|}{\mathscr{I}}}+\left(\frac{L_{, \alpha}}{2 m U_{0}}+\frac{M_{, \omega}}{2 \mathscr{I}}\right) .
\end{gathered}
$$



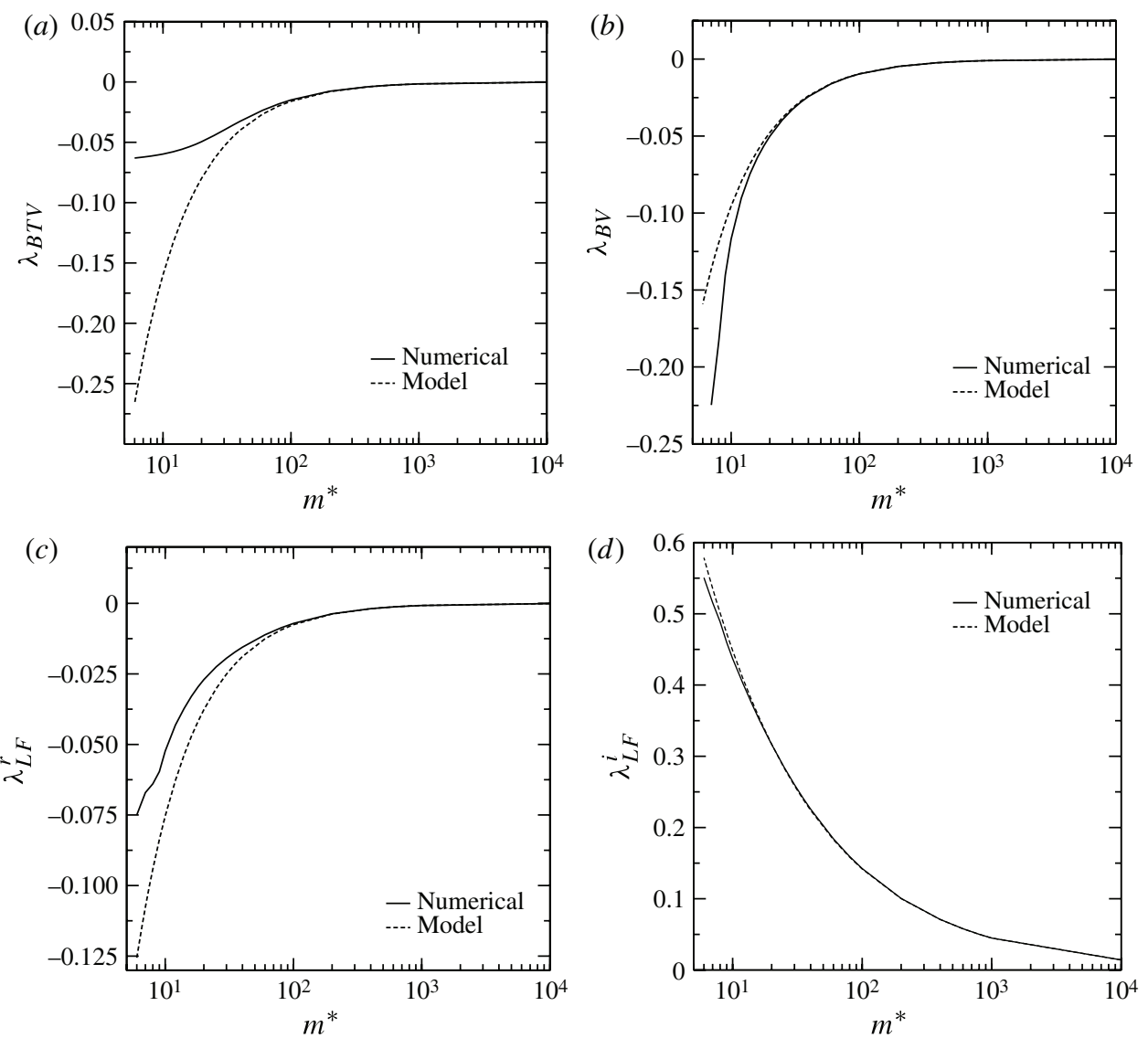

FIGURE 6. Variations of the eigenvalues in the flat plate problem with the mass ratio $m^{*}$ for $R e=30$ (lin-log scale). Comparison between results of the present numerical approach (solid lines) and predictions of the quasi-static model (dashed lines).

For most geometries of interest (with the noticeable exception of a rod with a square cross-section considered in $\S 6$ ), these four modes were found to be damped whatever the Reynolds number.

The eigenvalues computed through this quasi-static approach are compared with those obtained using the full numerical approach described in the previous section in figure 6. For $m^{*}=O\left(10^{2}\right)$ or higher, the two curves coincide for all four eigenvalues. The imaginary part $\lambda_{L F}{ }^{i}$ of $\lambda_{L F}$ is even well predicted by the quasi-static model throughout the range $m^{*}>8$.

The physical meaning of the aerodynamic modes was discussed in FAM. The first of them is symmetric and associated with a real, negative eigenvalue $\lambda_{B T V}$. This eigenvalue can be retrieved directly from (4.2); its interpretation is that, if the body velocity is higher (respectively, lower) than the equilibrium value $U_{0}$, the body experiences a larger (respectively, smaller) drag, which drives it back to the velocity $U_{0}$. This is why we call this mode 'back to terminal velocity' (BTV). The other three eigenvalues are associated with antisymmetric modes. One of them $\left(\lambda_{B V}\right)$ is real, negative and corresponds to a motion in which the body path comes back to the vertical while the body short axis (corresponding to the $\boldsymbol{x}_{b}$ direction) remains 

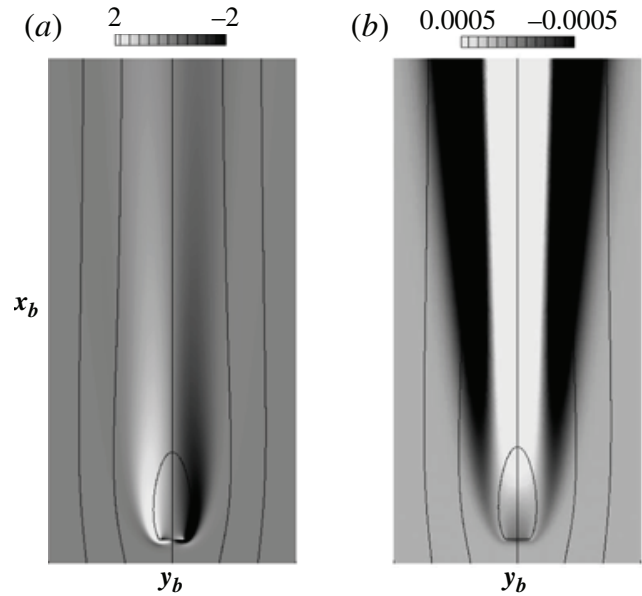

FIGURE 7. Structure of the two real 'aerodynamic' eigenmodes for a flat plate with $m^{*}=10^{4}$, $R e=30$ : $(a)$ 'back to terminal velocity' (BTV) mode; and (b) 'back to vertical' (BV) mode. The conventions used in the greyscale are similar to those of figure 5.

aligned with its path. We call this mode 'back to vertical' (BV). The leading-order approximation of the corresponding eigenvalue can be obtained directly from (4.3) by neglecting the two contributions provided by the lift force. The last two eigenvalues form a pair of complex conjugates, indicating an oscillating mode, the frequency of which is significantly smaller than that of the VK1 mode. For this reason, this mode, which can be interpreted as an oscillation of the body inclination along a nearly vertical path, is called 'low-frequency' (LF). The leading-order approximation of the corresponding eigenvalue can be retrieved directly from (4.4) by assuming $\alpha \approx \theta$ (hence $\gamma \approx 0$ ) and retaining only the component of the torque proportional to the incidence angle $\alpha$. Note, however, that, if the plate is initially vertical rather than horizontal, the coefficient $M_{, \alpha}$ is positive whatever $R e$ and (4.7) has to be replaced by $\lambda_{L F} \approx \pm \sqrt{M_{, \alpha} / \mathscr{I}}+L_{, \alpha} / 2 m U_{0}+M_{, \omega} / 2 \mathscr{I}$. Hence in this case the eigenvalues $\lambda_{L F}$ are real and no longer describe an oscillating mode. One of them is always positive (we checked that point using the quasi-static approach), indicating that this situation is always linearly unstable.

The structure of these 'aerodynamic' modes is illustrated in figures 7 and 8 . The real modes BTV and BV are displayed in the whole domain (figure $7 a, b$ ). Owing to obvious symmetry properties, the symmetric BTV mode has an antisymmetric vorticity distribution (just as the base flow), while the reverse is true for the antisymmetric BV mode. The complex LF mode is displayed in figure $8(a-c)$. Note that the maximum of the vorticity in figures 7 and 8 is much lower than that reached in the 'fluid' modes VK1 and VK2 displayed in figure $5(b, c)$. Since all three 'aerodynamic' modes have been normalized in such a way that $\hat{\theta}$ is unity, this feature indicates that the body motion and the vortical motions in the wake play an equal role in the dynamics of these modes. This is in stark contrast with what we observed for the 'fluid' modes. The BV mode (figure $7 b$ ) exhibits tiny values of vorticity, indicating that modifications in the wake play a negligible role in its dynamics. This is in line with the fact that the leading-order expression of $\lambda_{B V}$ can be obtained by simply considering that the body is subjected to the mean drag $-D_{0} \boldsymbol{x}_{a}$ and to the buoyancy-corrected weight $(\rho A-m) g \boldsymbol{x}_{0}$, which have the same magnitude but slightly different orientations. 

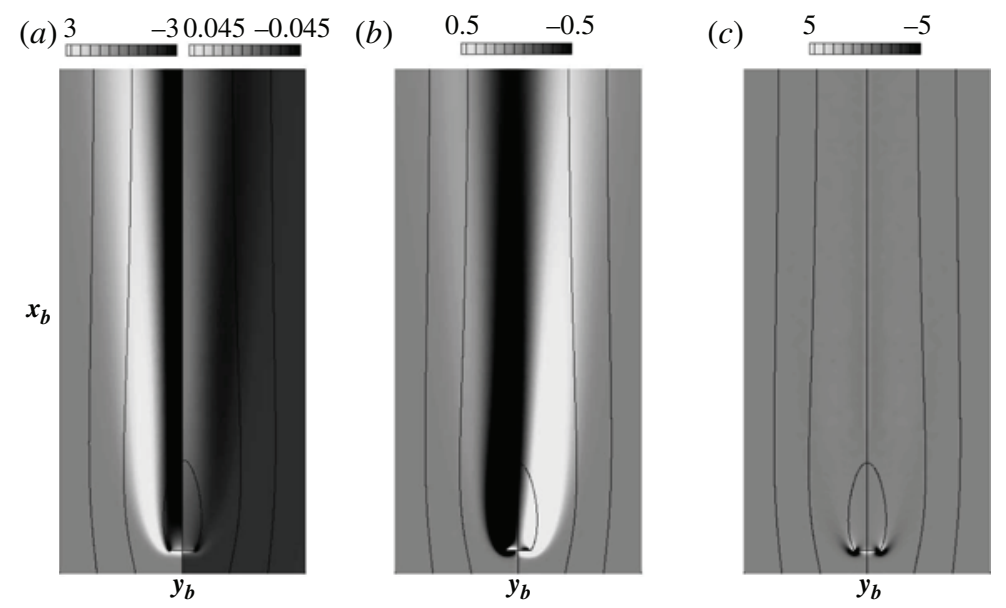

FIGURE 8. Structure of the complex 'aerodynamic' eigenmode LF (normalized by $\hat{\theta}$ ) for a flat plate with $m^{*}=10^{4}, R e=30$ : $(a)$ vorticity component $\hat{\omega} \cdot z$ of the LF mode; $(b)$ total vorticity $\boldsymbol{\omega} \cdot \boldsymbol{z}=\left(\boldsymbol{\omega}_{0}+\epsilon \hat{\boldsymbol{\omega}}\right) \cdot z$ with $\epsilon=0.1$; and $(c)$ real part of the absolute vorticity $\hat{\boldsymbol{\omega}}_{a b s} \cdot \boldsymbol{z}$ defined by (4.8). The left (respectively, right) side of the first two panels corresponds to the real (respectively, imaginary) contribution of the mode. The conventions used in the greyscale are similar to those of figure 5.

The structure of the LF mode deserves further explanation. Observations of the vorticity associated with this mode in the body frame of reference (figure $8 a$ ) indicate that the real and imaginary parts strongly differ both in structure and in amplitude; indeed, the imaginary part had to be magnified by a factor of 100 to reach the same range of iso-levels as the real part. This real part extends far downstream in the wake and consists of a central band of negative vorticity surrounded by two bands of positive vorticity. To better understand the significance of this structure, figure $8(b)$ displays the total vorticity $\boldsymbol{\omega} \cdot z=\left(\boldsymbol{\omega}_{0}+\epsilon \hat{\boldsymbol{\omega}}\right) \cdot z$ (where $\boldsymbol{\omega}_{0}=\nabla \wedge \boldsymbol{V}_{0}$ and $\left.\hat{\boldsymbol{\omega}}=\nabla \wedge \hat{\boldsymbol{v}}\right)$ by superposing the base-flow vorticity displayed in figure $5(a)$ onto the perturbation depicted in the left part of figure 8(a) (with an arbitrary amplitude $\epsilon=0.1$ ). The resulting flow has the same structure as the base flow, except that the wake is now tilted in the clockwise direction. This suggests that the banded structure observed in figure $8(a)$ is an indication that the wake develops in a direction that differs from that of the base flow, or, in other words, that the incidence angle associated with this LF mode is significant. To reinforce the validity of this interpretation, it is useful to re-express the vorticity associated with the eigenmode in the absolute reference frame, say $\hat{\boldsymbol{\omega}}_{a b s}$. The change of frame leads to the relation

$$
\hat{\boldsymbol{\omega}}_{a b s} \cdot z=\hat{\boldsymbol{\omega}} \cdot z+\hat{\theta}\left\{-x \partial_{y}\left(\boldsymbol{\omega}_{0} \cdot z\right)+y \partial_{x}\left(\boldsymbol{\omega}_{0} \cdot z\right)\right\}+2 \hat{\omega} .
$$

The real part of $\hat{\boldsymbol{\omega}}_{a b s} \cdot \boldsymbol{z}$ is displayed in figure $8(c)$. As can be seen, the banded structure initially visible in figure $8(a)$ has completely disappeared, indicating that the body motion induced by this mode has virtually no effect on the wake when the latter is considered in the actual direction along which it develops.

In figure $8(a)$, it may be noticed that the background level of the imaginary part of $\hat{\omega}$ is non-zero. Again, this is associated with the fact that the reference frame used to define $\hat{\boldsymbol{\omega}}$ is not inertial. Since the absolute vorticity $\hat{\boldsymbol{\omega}}_{\text {abs }}$ has to tend to zero far from the body, (4.8) implies that $\hat{\boldsymbol{\omega}} \cdot z \rightarrow-2 \hat{\omega}=-2 \lambda \hat{\theta}$ for large $\|\boldsymbol{r}\|$. Hence, at large 


\begin{tabular}{lccccccccc}
\hline$m^{*}$ & $R e$ & $\begin{array}{c}\text { Mode } \\
\text { name }\end{array}$ & $S t$ & $\begin{array}{c}|\hat{\boldsymbol{\omega}} \cdot z| \\
(1.5,0)\end{array}$ & $\kappa$ & $|\hat{\gamma}|$ & $\phi_{\hat{\gamma}}$ & $|\hat{\alpha}|$ & $\phi_{\hat{\alpha}}$ \\
10000 & 30 & VK1 & 0.104 & 34232.1 & 159891 & 0.174 & $-\pi / 3.1$ & 0.92 & $-\pi / 20$ \\
& & BV & - & 0.00019 & 0.008 & 0.999 & 0 & $6 \times 10^{-5}$ & 0 \\
& LF & 0.002 & 3.42044 & 0.0024 & 0.005 & $-\pi / 2$ & 0.999 & $-\pi / 563$ \\
& & & & & & & & \\
10000 & 73 & VK1 & 0.080 & 35698.7 & 1733700 & 0.109 & $-\pi / 3$ & 0.949 & $-\pi / 31.8$ \\
& VK2 & 0.089 & 30569 & 950138 & 0.119 & $-\pi / 2.7$ & 0.96 & $-\pi / 27.5$ \\
& BV & - & 0.00008 & 0.0055 & 0.999 & 0 & $7 \times 10^{-5}$ & 0 \\
& LF & 0.002 & 1.75843 & 0.0036 & 0.005 & $-\pi / 2$ & 0.999 & $-\pi / 570$
\end{tabular}

TABLE 3. Numerical values reached by various classification criteria for the antisymmetric eigenmodes of a heavy flat plate with $m^{*}=10^{4}$. The selected criteria are the Strouhal number, the modulus of the vorticity $\hat{\boldsymbol{\omega}}$ at the location $(x=1.5, y=0)$, the kinetic energy ratio $\kappa$, the modulus and phase of the components of the eigenmode associated with the slope of the path $\hat{\gamma}$, and the incidence angle $\hat{\alpha}$, respectively.

distance from the body, $\hat{\boldsymbol{\omega}} \cdot z$ is mostly an imaginary quantity since $\lambda_{L F} \approx \lambda_{L F}^{i}$ (see figure 6) and $\hat{\theta}=1$.

To summarize, both the 'banded' structure of the real part of the vorticity associated with the LF mode and the non-zero background level of its imaginary part are artifacts arising as a result of the use of a system of axes that is not aligned with the actual direction of the flow. These artifacts disappear when considering the absolute vorticity in the aerodynamic axes. However, they do provide a useful visual criterion for detecting that the body significantly drifts laterally and rotates. We found this property useful for describing the structure of the modes observed in other ranges of the $\left(m^{*}, \chi, R e\right)$ parameters. This is why in what follows we keep on representing the structure of the modes using the vorticity $\hat{\omega}$ expressed in the body reference frame.

\subsubsection{Classification criteria}

Up to now we have distinguished the 'fluid' component of the eigenmodes from their 'solid' counterpart thanks to the visual criterion based on the maximum of the vorticity, and used the structure of the vorticity in the relative reference frame to identify modes containing a significant incidence and rotation. We also looked for more quantitative criteria to investigate the nature of the eigenmodes. Table 3 presents the values reached by a number of such criteria when applied to the antisymmetric modes encountered so far for $m^{*}=10^{4}$.

A natural idea is to select as such a criterion the maximum level of some characteristics of the flow component associated with the eigenmode, either the velocity $\hat{\boldsymbol{v}}$, the pressure $\hat{p}$, or the vorticity $\hat{\boldsymbol{\omega}}$. However, all of them reach very large levels far downstream in the wake, a feature associated with the convective nature of the instability in the far wake. In addition, the pressure and vorticity are singular at the corners of the plate, making such intuitive criteria useless. As a consequence, we chose to retain the norm of the vorticity component at a given point arbitrarily selected within the wake, namely $|\hat{\boldsymbol{\omega}} \cdot \boldsymbol{z}|(1.5,0)$. As can be seen in the fifth column of table 3, this criterion reaches large values for the 'fluid' modes VK1 and VK2, indicating that these modes are associated with a weak displacement of the body compared to the intensity of the vortical activity in the wake (keep in mind that all antisymmetric eigenmodes are normalized by assuming $\hat{\theta}=1$ ). On the other hand, 
this criterion reaches $O(1)$ or lower values for the 'aerodynamic' modes BV and LF, suggesting a stronger coupling between the fluid and the body. This criterion also allows us to distinguish the LF mode, for which $|\hat{\boldsymbol{\omega}} \cdot z|(1.5,0)$ is of $O(1)$, from the BV mode, for which this quantity is much smaller. Another intuitive criterion is the ratio of the kinetic energies respectively associated with the fluid and body motion. However, the first of these energies is infinite owing to the unbounded nature of the flow. Nevertheless, the kinetic energy can be computed within a fixed bounded domain surrounding the body, say $\Gamma$, whose outer boundary is fixed arbitrarily. We defined $\Gamma$ as the dashed rectangle that surrounds the body in figure 2 , i.e. $\Gamma=[-1 ; 4] \times[0 ; 1.5]$ in the $\left(\boldsymbol{x}_{b}, \boldsymbol{y}_{b}\right)$ axes. Hence we define the kinetic energy ratio as

$$
\kappa=\frac{\int_{\Gamma} \rho|\hat{\boldsymbol{v}}|^{2} \mathrm{~d} \Gamma}{m\left|\hat{u_{y}}\right|^{2}+\mathscr{I}|\hat{\omega}|^{2}} .
$$

The sixth column in table 3 shows the values of $\kappa$ corresponding to the various modes. In contrast with the previous criterion, $\kappa$ does not allow us to distinguish between the BV and LF modes. However, it discriminates better between the 'fluid' and 'aerodynamic' modes, which are associated with highly contrasted values of the kinetic energy ratio, a useful feature in what follows. The last four columns in table 3 correspond to the modulus and phase of the components of the eigenmodes associated with the slope of the path, $\hat{\gamma}$, and the incidence angle, $\hat{\alpha}$, respectively (still with the normalization $\hat{\theta}=1$ ). The reported values indicate that $\hat{\gamma} \approx \hat{\theta}$ for the BV mode, whereas $\hat{\alpha} \approx \hat{\theta}$ for the LF mode, as predicted by the quasi-static approach of FAM. On the other hand, the values of $\hat{\gamma}$ show that the incidence $\hat{\alpha}=\hat{\theta}-\hat{\gamma}$ is close to the inclination $\hat{\theta}$ for 'fluid' modes. Nevertheless, these two angles are not asymptotically close since the slope $\hat{\gamma}$ is small but not negligible.

\subsection{Parametric study for $O(1)$ mass ratios}

We are now in position to discuss the results provided by the parametric study that we carried out throughout the whole range of mass ratios $m^{*}$. No unstable modes were found among the symmetric eigenmodes, so we only document the evolution of the antisymmetric modes. We first show how the branches of the unstable modes evolve with the Reynolds number for several values of $m^{*}$ and then synthesize the results by presenting a stability diagram in the $\left(m^{*}, R e\right)$ plane. Then we describe the spatial structure of the computed unstable modes.

\subsubsection{Study of the branches of unstable eigenvalues as function of Re}

As we saw in figure 4, in the large- $m^{*}$ limit the problem exhibits two pairs of complex eigenmodes of 'fluid' nature, which are successively destabilized as the Reynolds number increases, plus four other modes, which are always damped. We now explore how this situation evolves as the mass ratio $m^{*}$ decreases.

Figure 9 shows the real part of the computed eigenvalues in the range of Reynolds number $\operatorname{Re} \in[19,100]$, for several values of $m^{*}$. The case $m^{*}=10$ (figure $9 a$ ) remains qualitatively similar to the large- $m^{*}$ situation, with two branches crossing the real axis (for $\operatorname{Re} \approx 30$ and $R e \approx 70$, respectively). A third, always damped, branch is also observed (this branch roughly follows the asymptotic prediction for the 'aerodynamic' LF mode). Down to $m^{*}=4.75$, the configuration of the branches remains qualitatively similar. Note that the first branch becomes unstable earlier. On the other hand, the second branch becomes almost tangent to the real axis for $R e \approx 60$ but the actual threshold is delayed up to $R e \approx 80$. In the interval $m^{*} \in[4.5,4.75]$ (figure $9 a$ ), the 

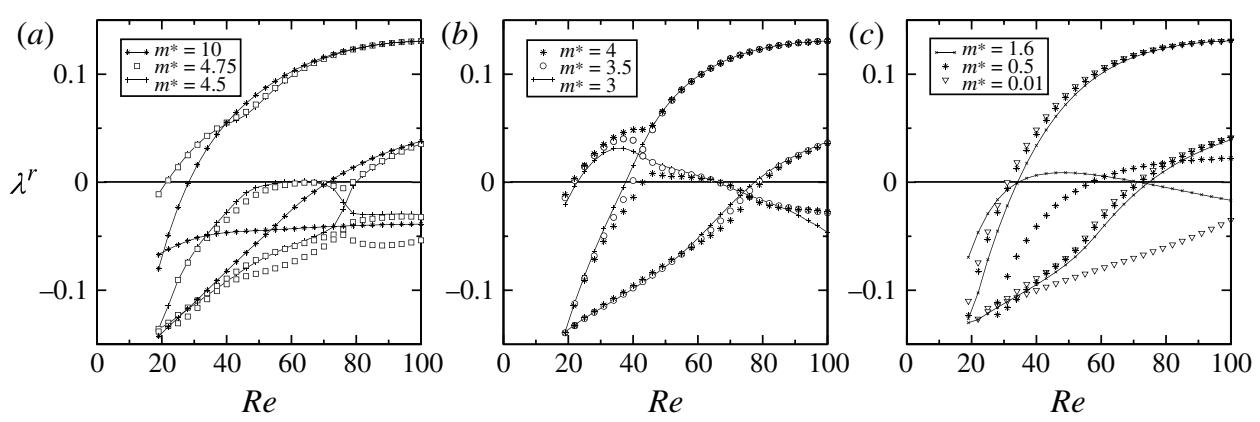

FIGURE 9. Real part $\lambda^{r}$ of the eigenvalues of the antisymmetric modes as a function of $R e$ for a flat plate with several different values of $m^{*}:(a) 10 \geqslant m^{*} \geqslant 4.5$; (b) $4 \geqslant m^{*} \geqslant 3$; and (c) $1.6 \geqslant m^{*} \geqslant 0.01$.

second and third branches cross and exchange their identity. The thresholds are not affected by this switch, but the second threshold now occurs on the continuation of the branch that was formerly the most damped one, while the second branch goes down towards negative values after approaching the real axis from below. Decreasing the mass ratio down to $m^{*}=4$ (figure $9 b$ ), it can be observed that the second branch now becomes unstable within a bounded range of Reynolds number, with a destabilization point at $R e \approx 40$ and a restabilization point at $R e \approx 65$. Further decreasing $m^{*}$ down to $m^{*}=3.5$, the next event to occur is a crossing between the first and second branches, which exchange their identity. After this transition, the restabilization point at $R e \approx 60$ occurs on the first branch, while values located on the second branch increase monotonically with $R e$. Still decreasing $m^{*}$ (figure $9 c$ ), the destabilization point of the first branch is delayed towards higher Reynolds numbers, until a crossing occurs with the second branch for $m^{*} \approx 1.6$. Hence, for lower $m^{*}$, the first destabilization point occurs along the second branch. At the same time, the restabilization point is also delayed and occurs after the third destabilization point for $m^{*} \leqslant 1$.6. For small values of $m^{*}$, e.g. $m^{*}=0.01$, we are left with a simpler situation where two unstable branches of modes successively become unstable (for $R e \approx 31.5$ and $R e \approx 70.9$, respectively), while the third branch is associated with eigenvalues with a negative real part, so that the third threshold is rejected to much higher Reynolds numbers and does not appear in the figure any more.

\subsubsection{Neutral curves in the $\left(m^{*}, R e\right)$ plane and corresponding Strouhal numbers}

Figure 10(a) shows the neutral curves (defined as the locations where an eigenvalue crosses the real axis) in the $\left(m^{*}, R e\right)$ plane. As a consequence of the complex branch reorganization that we have just described, three neutral curves are encountered. The first two (denoted as $B 1$ and $B 2$ ) are associated with the destabilization of an eigenmode and exist whatever $m^{*}$. The third curve (denoted as B3) only exists for $m^{*}<4.75$ and displays both a destabilization branch (dashed-dotted line) and a restabilization branch (dotted line). For large mass ratios, curves $B 1$ and $B 2$ can be identified with the onset of the VK1 and VK2 'fluid' modes. However, as the notion of 'fluid' versus 'aerodynamic' mode only makes sense for large $m^{*}$, we prefer to abandon this terminology when commenting the behaviours found along the neutral curves and simply refer to these curves as $B 1, B 2$ and $B 3$. As was seen in the previous subsection, the latter exchange their identity and respective position several times, making it impossible to classify them rationally. 

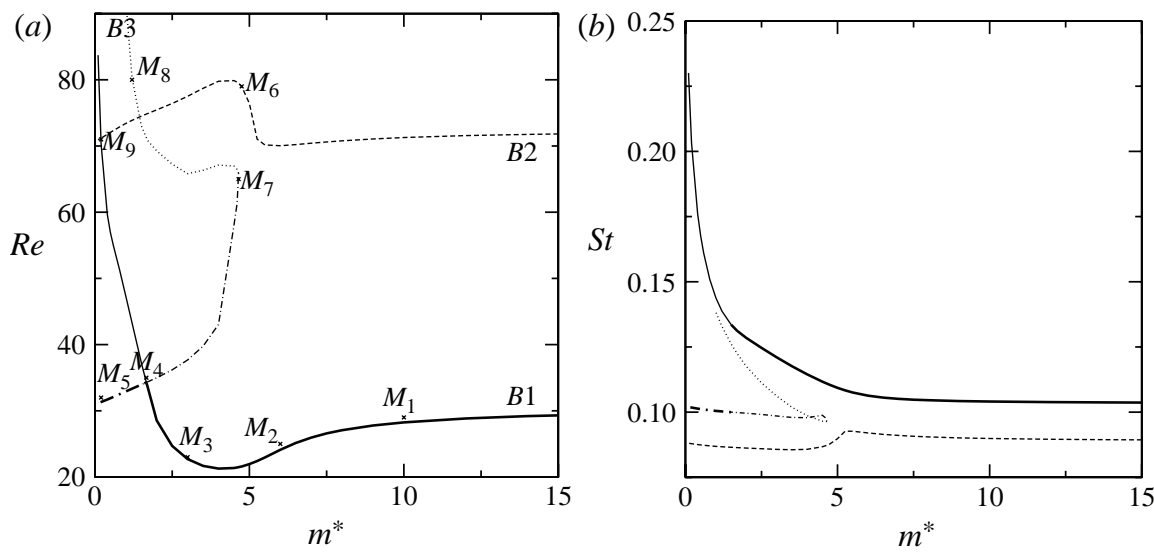

FIGURE 10. (a) Neutral curves in the $\left(m^{*}, R e\right)$ plane for a flat plate. The points denoted as $M_{i}$ identify modes that will be described later. (b) Strouhal number associated with the neutral curves. In both panels the three curves are the branches $B 1$ (solid line), $B 2$ (dashed line) and $B 3$ (dash-dotted and dotted lines). The marginal Reynolds number $\operatorname{Re}_{c}\left(m^{*}\right)$ and the marginal Strouhal number $S t_{c}\left(m^{*}\right)$ associated with the first destabilization are indicated with a thicker line.

From a practical point of view, the most important result displayed in figure 10 is the lowest marginal curve, which defines the critical Reynolds number $\operatorname{Re}_{c}\left(m^{*}\right)$ at which the body path is first destabilized (this curve is marked with a thicker line in figure $10 a$ ). For large $m^{*}$, this marginal curve follows the $B 1$ branch, and in the limit $m^{*} \gg 1$ the threshold is in agreement with that found for a fixed plate, namely $\operatorname{Re}_{c}(\infty)=30.5$. When the mass ratio decreases, it can be observed that the critical Reynolds number first decreases and reaches a minimum for $m^{*}=4$, where $\operatorname{Re}_{c}=21.2$. The critical Reynolds number then increases again to reach $\operatorname{Re}_{c}=34.3$ for $m^{*}=1.6$. At this point, a crossing occurs with the branch $B 3$. The marginal stability curve then continues along the latter branch, and the critical Reynolds number decreases to reach $R e_{c} \approx 30.6$ in the limit of very light bodies $\left(m^{*} \approx 0\right)$.

Figure 10(b) shows the Strouhal numbers found along the three neutral curves. The critical Strouhal number associated with the first destabilization, denoted as $\operatorname{St}_{c}\left(m^{*}\right)$, is indicated with a thicker line. When the mass ratio decreases from $m^{*}=\infty$ to $m^{*}=1.6$, $S t_{c}\left(m^{*}\right)$ increases monotonically from $S t_{c}=0.103$ (in agreement with the value found for a fixed plate) to $S t_{c}=0.132$. Then, as the marginal curve switches from branch $B 1$ to branch $B 3$, the corresponding Strouhal number abruptly jumps to $S t_{c}=0.099$. Further decreasing $m^{*}$, the marginal Strouhal number weakly increases to reach the value $S t_{c}=0.102$ in the limit $m^{*}=0$.

It is noteworthy that the threshold and frequency exhibit the same values in the limit of both small and large mass ratios. For instance, $R e_{c} \approx 31$ and $\lambda^{i}=0.643$ for $m^{*}=10^{-4}$, while $R e_{c} \approx 30.6$ and $\lambda^{i}=0.649$ for $m^{*}=10^{4}$. We do not have a rational explanation for this coincidence, which is most likely to be fortuitous.

\subsubsection{Structure of the unstable modes}

We now turn to the structure of the eigenmodes found along the various neutral curves. For this, a selection of eigenmodes corresponding to the points labelled $M_{i}$ in figure $10(a)$ is displayed in figures 11 and 12. The corresponding values of $R e$ and 


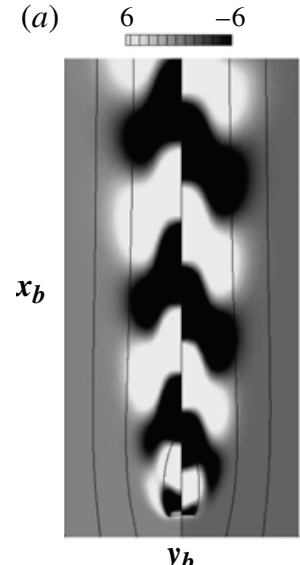

$y_{b}$

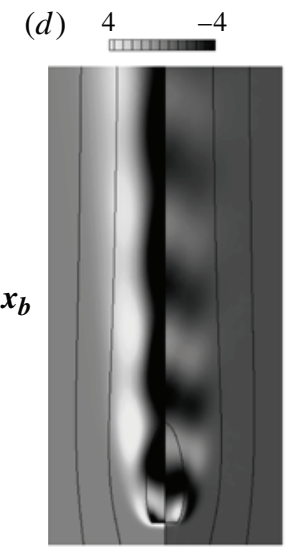

$y_{b}$ (b) 3

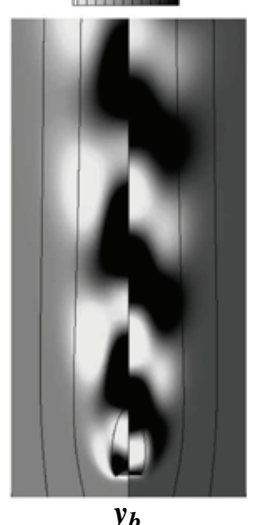

(e)

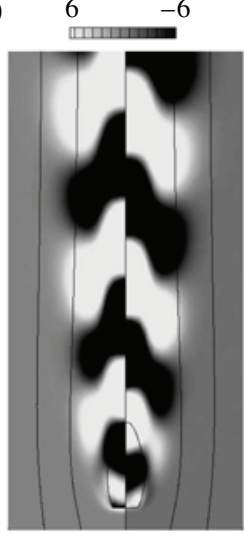

(c) 3

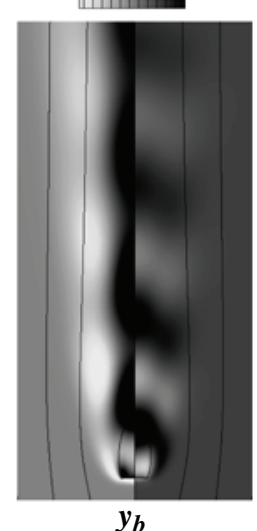

(f) 9

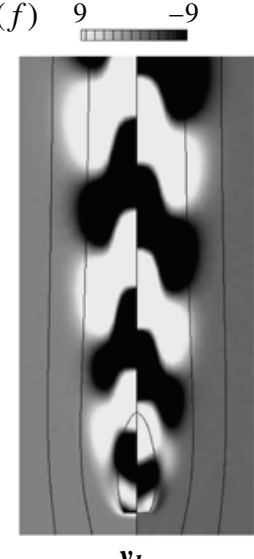

FIGURE 11. Structure of some eigenmodes taken along the marginal stability curve for a flat plate: $(a)$ mode $M_{1}$ with $m^{*}=10, R e=29 ;(b)$ mode $M_{2}$ with $m^{*}=6, R e=25 ;(c)$ mode $M_{3}$ with $m^{*}=3, R e=23 ;(d)$ mode $M_{4}$ (on branch $B 1$ in figure $10 a$ ) with $m^{*}=1.665, R e=35$; (e) mode $M_{4}^{\prime}$ (on branch $B 3$ in figure $10 a$ ) with $m^{*}=1.665, R e=35$; and $(f)$ mode $M_{5}$ with $m^{*}=0.1, R e=31$. The modes are displayed with iso-levels of the vorticity component using the same conventions as in figure 5 .

$m^{*}$ are given in table 4, together with the value of the various quantitative criteria introduced in $\S 4.1 .3$.

Figure 11 gathers eigenmodes encountered along the marginal curve, which successively corresponds to the $B 1$ (respectively, B3) branch for $m^{*}$ larger (respectively, smaller) than 1.665 . For $m^{*}=10$ (mode $M_{1}$, figure $11 a$ ) the structure is very similar to that of the VK1 'fluid' mode obtained in the limit of large $m^{*}$ (compare with figure $5 b$ ). However, it may be noticed that the iso-vorticity levels now reach a much lower value, indicating that the displacement of the body has become significant. When $m^{*}$ decreases down to $m^{*}=6$ (respectively, $m^{*}=3$ ), the spatial structure displayed in figure $11(b)$ (respectively, 11c) changes with the occurrence of a banded structure akin to the one observed for the 'aerodynamic' LF mode (see figure 8); the background level of the imaginary part of the vorticity also increases (see the increase of the average grey level in the right half of figure $11 a-c$ ). As previously 


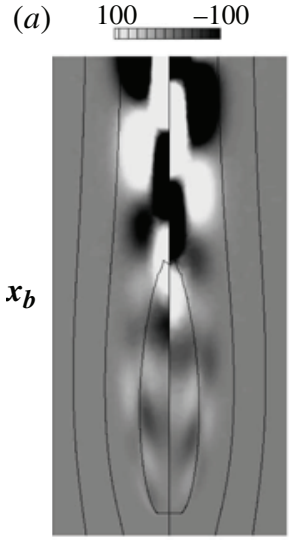

$y_{b}$ (b) 50

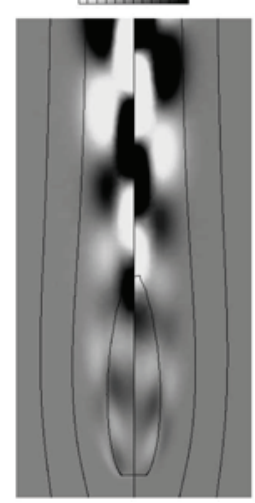

$y_{b}$ (c)

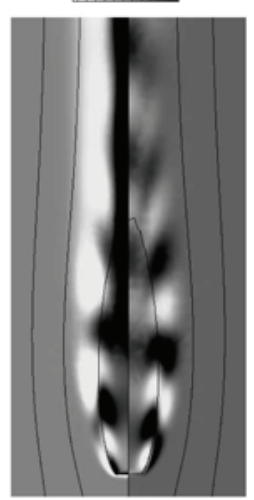

$y_{b}$ (d) 7

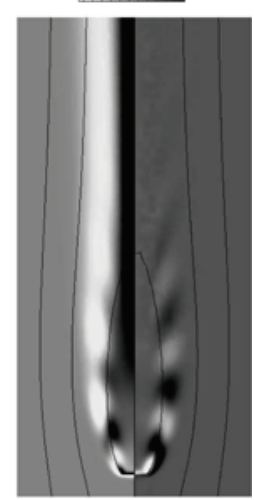

$y_{b}$

FIGURE 12. Structure of some eigenmodes taken along the higher neutral curves for a flat plate: $(a)$ mode $M_{6}$ with $m^{*}=4.75, R e=79 ;(b)$ mode $M_{7}$ with $m^{*}=4.65, R e=65$; (c) mode $M_{8}$ with $m^{*}=1.2, R e=80$; and $(d)$ mode $M_{9}$ with $m^{*}=0.2, \operatorname{Re}=71$. The modes are displayed with iso-levels of the vorticity component using the same conventions as in figure 5 .

\begin{tabular}{lccccccccc}
\hline$m^{*}$ & $R e$ & $\begin{array}{c}\text { Mode } \\
\text { label }\end{array}$ & $S t$ & $\begin{array}{c}|\hat{\omega} \cdot z| \\
(1.5,0)\end{array}$ & $\kappa$ & $|\hat{\gamma}|$ & $\phi_{\hat{\gamma}}$ & $|\hat{\alpha}|$ & $\phi_{\hat{\alpha}}$ \\
10 & 29 & $M_{1}$ & 0.104 & 18.2564 & 26.5346 & 0.198 & $-\pi / 2.7$ & 0.937 & $-\pi / 16.1$ \\
6 & 25 & $M_{2}$ & 0.106 & 4.53221 & 13.1324 & 0.21 & $-\pi / 2.5$ & 0.954 & $-\pi / 14.9$ \\
3 & 23 & $M_{3}$ & 0.121 & 2.92701 & 16.594 & 0.248 & $-\pi / 2.3$ & 0.985 & $-\pi / 12.5$ \\
1.665 & 35 & $M_{4}$ & 0.131 & 4.21929 & 30.2515 & 0.345 & $-\pi / 2.2$ & 1.016 & $-\pi / 9.1$ \\
& 35 & $M_{4}^{\prime}$ & 0.100 & 14.5511 & 179.513 & 0.322 & $-\pi / 1.8$ & 1.114 & $-\pi / 11$ \\
0.1 & 31 & $M_{5}$ & 0.102 & 24.0528 & 5177.12 & 0.287 & $-\pi$ & 1.287 & 0 \\
0.001 & 31 & & 0.102 & 24.0277 & 531161 & 0.267 & $-\pi / 0.96$ & 1.266 & $\pi / 128$ \\
4.75 & 79 & $M_{6}$ & 0.088 & 4.14834 & 97.3161 & 0.234 & $-\pi / 2.2$ & 0.988 & $-\pi / 13$ \\
4.65 & 65 & $M_{7}$ & 0.097 & 4.02627 & 47.8136 & 0.241 & $-\pi / 2.3$ & 0.98 & $-\pi / 12.9$ \\
1.2 & 80 & $M_{8}$ & 0.133 & 2.71951 & 1810.05 & 0.474 & $-\pi / 2.1$ & 1.062 & $-\pi / 6.8$ \\
0.2 & 71 & $M_{9}$ & 0.203 & 2.9554 & 147.007 & 0.948 & $-\pi / 1.8$ & 1.503 & $-\pi / 4.7$
\end{tabular}

TABLE 4. Values of various classification criteria for a selection of eigenmodes located close to the marginal stability curves in the case of a flat plate. The nomenclature employed for the criteria is similar to that defined in table 3 and $\S 4.1 .3$. The labelled modes are displayed in figures 10-12.

discussed, both features are indicative of a significant misalignment of the body with respect to its path. As can be seen visually through the iso-vorticity levels and more quantitatively through the values of $|\hat{\boldsymbol{\omega}} \cdot z|(1.5,0)$ displayed in table 4 , this is accompanied by a decrease of the ratio of the 'fluid' and 'solid' parts of the eigenmode, which highlights the strong fluid-solid coupling that starts to take place for such values of $m^{*}$.

As we already noticed, a crossing between two neutral curves occurs for $m^{*}=1.665$. Accordingly, two modes are simultaneously neutral; they are displayed in figure $11(d, e)$. As can be observed, these two modes have a rather different structure. The one belonging to branch $B 1$ in figure $10(a)$ is qualitatively similar to those found 
on the same branch at slightly higher values of $m^{*}$. In contrast, the mode belonging to branch $B 3$ is much more akin to the VK1 'fluid' mode existing at much larger values of $m^{*}$. As $m^{*}$ further decreases, the structure of the eigenmode does not evolve much along the marginal curve (which now corresponds to the $B 3$ branch), as revealed by figure $11(f)$ for $m^{*}=0.1$. However, changes in the phases $\phi_{\hat{\gamma}}$ and $\phi_{\hat{\alpha}}$ may be noticed (see table 4), and $\hat{\alpha}$ and $\hat{\gamma}$ become almost in phase with $\hat{\theta}$ for $m^{*}=0.1$.

Finally, figure 12 displays a few samples of the eigenmodes encountered along the higher neutral curves. The mode $M_{6}$ shown in figure 12(a) belongs to the branch $B 2$ and its structure is similar to that of the VK2 'fluid' mode found on the same branch in the limit of large $m^{*}$ (compare with figure $5 c$ ). The mode $M_{7}$ displayed in figure $12(b)$ is the one found at the turning point on branch $B 3$; its structure is almost the same as that of the mode $M_{6}$. The last two modes $M_{8}$ and $M_{9}$ displayed in figure 12 are found on the restabilization branch of the neutral curve $B 3$ and on the low- $m^{*}$ part of the neutral curve $B 1$ after its crossing with branch $B 3$, respectively. Both have higher oscillation frequencies $\lambda^{i}$ compared to the previous modes (see the Strouhal numbers in table 4). Accordingly, their spatial structure is characterized by a shorter wavelength. Their real part also displays a banded structure indicative of a significant coupling between the fluid and the body.

\section{The rectangular plate with $\chi=8$}

Falling plates with an aspect ratio $\chi=8$ and either a rectangular or an elliptical cross-section have been investigated in several recent studies (Andersen et al. 2005a,b; Jin \& Xu 2008; Kolomenskiy \& Schneider 2010). In this section we consider this geometry in the case of a rectangular cross-section at zero incidence and describe the corresponding marginal stability curves as we did in the previous section. We then provide a few comparisons with available work.

\subsection{Results}

The situation revealed by the computations that we carried out in the case $\chi=8$ is actually quite similar to that we just described for a flat plate. In particular, in the limit of large mass ratios, 'fluid' modes of the VK1 and VK2 type and 'aerodynamic' modes of the BTV, BV and LF type are encountered, those of the latter series always being weakly damped. For smaller mass ratios, several branches of unstable modes exist and exchange their respective position and identity in a complicated way, which shares some similarities with, but also reveals some differences from, the thin plate case. We do not detail the whole process here but restrict ourselves to the mapping of the neutral curves and the description of the structure of a few modes found along the marginal stability curve.

Figure 13(a) displays the neutral curves in the $\left(m^{*}, R e\right)$ plane, while figure $13(b)$ shows how the Strouhal number corresponding to the various branches evolves. As in the thin plate case, three neutral curves coexist. The first two of them (labelled $B 1$ and $B 2$ ) correspond to the destabilization of an eigenmode, while the third (labelled B3) exhibits both a destabilization and a restabilization branch. Several noticeable differences with the thin plate case can be pointed out. The most salient of these is that no crossing exists in the lower part of the diagram, so that the instability threshold occurs along the same $B 1$ branch whatever $m^{*}$. Another difference is that the branch $B 3$ displays a small loop bounding a region of the $\left(m^{*}, R e\right)$ plane within which only one unstable mode exists. Figure 13(b) complements this description by displaying the corresponding Strouhal numbers. It may be noticed that $S t$ does not vary much along 

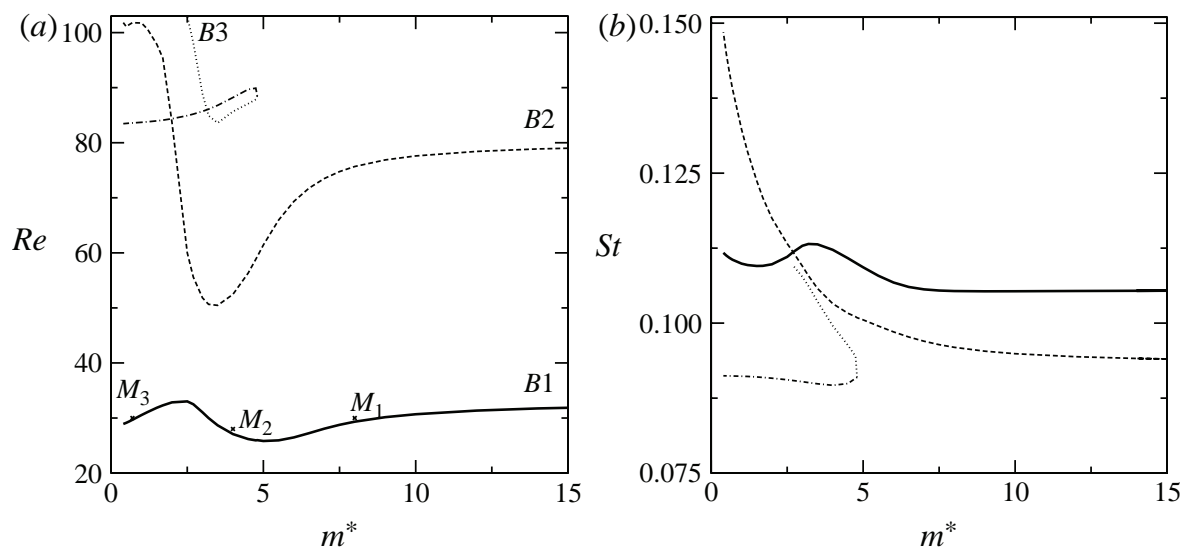

FIGURE 13. Stability of a rectangular plate with $\chi=8$ at zero incidence: $(a)$ neutral curves in the $\left(m^{*}, R e\right)$ plane; and $(b)$ associated Strouhal number. The same conventions are used as in figure 10.

the $B 1$ branch, staying in the range $0.108<S t<0.12$ throughout the whole range of $m^{*}$ reported in the figure.

Despite the structural differences observed between the neutral curves of figures $13(a)$ and 10(a), there are also noticeable similarities. In particular, the limit cases $m^{*} \gg 1$ and $m^{*} \ll 1$ behave similarly. That is, two unstable modes, which may again be identified with the VK1 and VK2 modes of the corresponding fixed body, are found in the $m^{*} \gg 1$ limit. In the low- $m^{*}$ limit, two unstable modes also exist, with critical Reynolds numbers falling in the same range as for the thin plate. The thresholds are slightly higher than in the thin plate case in both limits, since $R e_{c} \approx 30.3$ for $m^{*} \gg 1$ and $R e_{c} \approx 29$ for $m^{*} \ll 1$. A last noticeable similarity is the evolution of the instability threshold curve (thick line in figures $13 a$ and $10 a$ ) with $m^{*}$ : as the mass ratio decreases, the threshold Reynolds number first decreases (down to $R e=25.8$ for $m^{*}=5$ and $\chi=8$ ), then increases back (up to $R e=33$ for $m^{*}=2.5$ and $\chi=8$ ), before decreasing again. The fact that the second neutral curve bends downwards strongly for $m^{*} \approx 3$ indicates the proximity of a second mode, which could interact with the leading mode in this range of mass ratio.

The structure of the eigenmodes found along the marginal stability curve is displayed in figure 14; the corresponding values of the classification criteria are given in table 5. As $m^{*}$ decreases, the marginal modes successively display a VK1-like structure, then a modulated tail structure, before recovering a VK1-like structure for small $m^{*}$. This evolution is also qualitatively similar to that encountered in the thin plate case.

\subsection{Comparison with previous work}

Most available studies devoted to the free rise or fall of a rectangular plate have focused on the range $R e=O\left(10^{3}\right)$, which corresponds to the transition between fluttering and tumbling motions. Hence direct comparison with our work is limited. Kolomenskiy \& Schneider (2010) computed the free fall of plates with an elliptic cross-section of aspect ratio $\chi=8$ and a density ratio $\sim 2.7$ (which, using our notation, corresponds to a mass ratio $m^{*} \approx 0.26$ ) for three widely separated values of the Archimedes number, namely $A r \approx 7.1,71$ and 710 . Their results indicate that, after an initial transient, the path is steady and vertical in the former two cases, while the plate 


\begin{tabular}{cccccccccc}
\hline$m^{*}$ & $R e$ & $\begin{array}{c}\text { Mode } \\
\text { label }\end{array}$ & $S t$ & $\begin{array}{c}|\hat{\omega} \cdot z| \\
(1.5,0)\end{array}$ & $\kappa$ & $|\hat{\gamma}|$ & $\phi_{\hat{\gamma}}$ & $|\hat{\alpha}|$ & $\phi_{\hat{\alpha}}$ \\
8.0 & 30 & $M_{1}$ & 0.105 & 1.3233 & 31.80 & 0.214 & $-\pi / 3$ & 0.909 & $-\pi / 15.4$ \\
4.0 & 28 & $M_{2}$ & 0.112 & 1.4063 & 24.78 & 0.16 & $-\pi / 3.4$ & 0.914 & $-\pi / 22.3$ \\
0.7 & 30 & $M_{3}$ & 0.110 & 1.3887 & 592.3 & 0.299 & $-\pi / 18$ & 0.707 & $-\pi / 42$ \\
0.01 & 28.89 & & 0.114 & 1.436 & 36450 & 0.396 & $-\pi / 40$ & 0.609 & $-\pi / 60$
\end{tabular}

TABLE 5. Values of various classification criteria for a selection of eigenmodes located close to the marginal stability curves in the case of a rectangular plate with $\chi=8$. The nomenclature employed for the criteria is similar to that defined in table 3 and $\S 4.1 .3$. The labelled modes are displayed in figure 14.
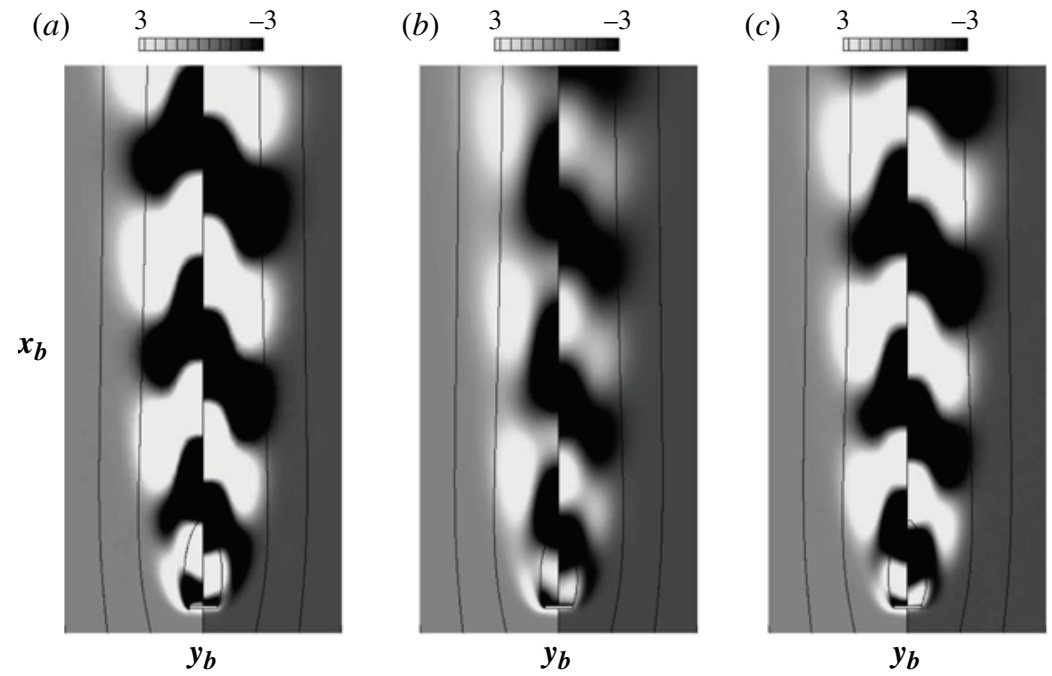

FIGURE 14. Structure of some eigenmodes of a rectangular plate with $\chi=8$ taken along the marginal stability curve: $(a)$ mode $M_{1}$ with $m^{*}=8, R e=30 ;(b)$ mode $M_{2}$ with $m^{*}=4, R e=28$; and (c) mode $M_{3}$ with $m^{*}=0.7, R e=30$. The modes are displayed with iso-levels of the vorticity component using the same conventions as in figure 5 .

first flutters and then tumbles for $A r=710$, which suggests that the onset of fluttering takes place in the range $71<A r<710$ (assuming that $C_{d} \approx 1$ in (2.13), this suggests a critical Reynolds number in the range $\left.100<R e_{c}<1000\right)$. These findings seem at odds with the present results since the latter predict a critical Reynolds number $\sim 29$ for the same value of $m^{*}$. However, the conclusion of Kolomenskiy \& Schneider (2010) regarding their results at $A r \approx 71$ relies on a visual examination of the path and they do not show any evidence that the wake is still stable. Full Navier-Stokes computations performed in our team in the same range of parameters (Auguste 2010) lead to a different conclusion, as they show that the converged state is characterized by a Von Kármán-like wake with almost no signature on the path. This conclusion agrees qualitatively with the present results, which reveal that in this range of $m^{*}$ the unstable eigenmode mostly manifests itself in the wake but not in the path. 

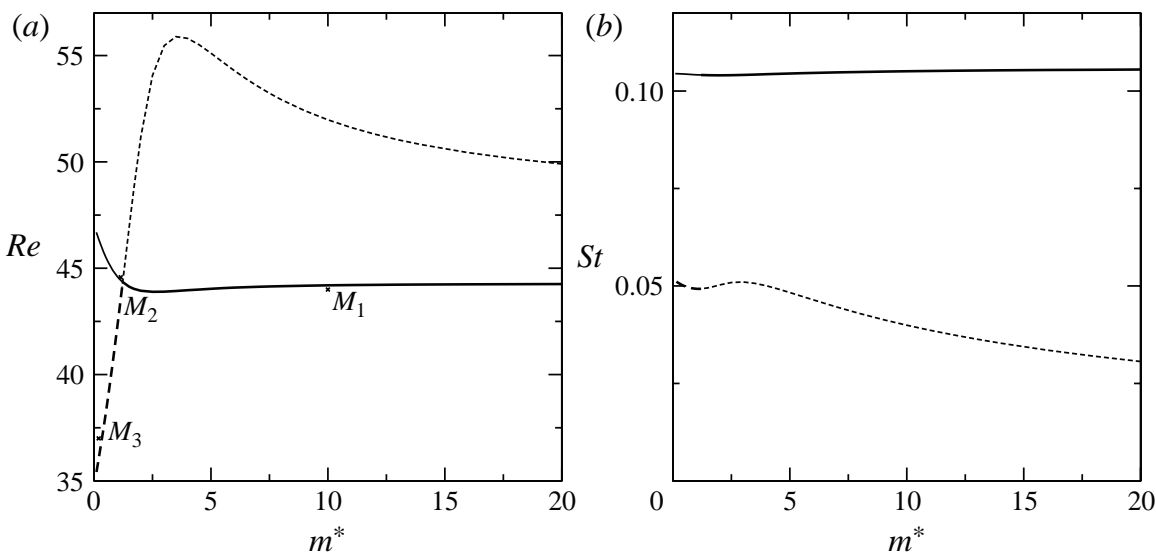

FIGURE 15. Stability of a square rod at zero incidence: $(a)$ neutral curves in the $\left(m^{*}, R e\right)$ plane; and $(b)$ associated Strouhal number. The same conventions are used as in figure 10.

\section{The square rod}

We finally consider the case of a rod with a square cross-section at zero incidence with respect to the incoming stream. In contrast to thinner plates, for which the quasi-steady model introduced in FAM predicts that the various 'aerodynamic' modes are damped whatever $R e$ in the large- $m^{*}$ limit, a low-frequency (LF) mode becoming unstable beyond $R e \approx 48$ is predicted in the case of a square rod. Interestingly, this threshold is very close to that corresponding to the onset of vortex shedding for a fixed rod, the latter occurring at $R e \approx 46$ (Sohankar, Norberg \& Davidson 1998; Lankadasu \& Vengadesan 2008). We refer to the associated 'fluid' mode as the VK1 mode in line with the terminology used in previous sections. It is worth noting that we explored the stability of a fixed square rod up to $R e \approx 150$ and did not find the existence of a secondary vortex shedding mode such as the VK2 mode identified for fixed plates with aspect ratios $\chi=10^{4}$ and $\chi=8$.

The mapping of the neutral curves in the $\left(m^{*}, R e\right)$ plane was achieved in the same way as with previous geometries. The results are shown in figure 15 along with the corresponding Strouhal numbers. Only two neutral curves are found to exist for this specific geometry. The first branch, denoted as $B 1$, coincides with the VK1 'fluid' mode in the large- $m^{*}$ limit and remains almost horizontal with $R e \approx 44.5$ over most of the $m^{*}$ range reported in the figure. The corresponding Strouhal number is also almost constant, with $S t \approx 0.104$, in agreement with the fixed-body value (Sohankar et al. 1998). The second branch, denoted as B2, coincides with the LF 'aerodynamic' mode in the large- $m^{*}$ limit. The corresponding Strouhal number is always lower by at least a factor of 2 compared with that of the VK1 mode. This Strouhal number increases as $m^{*}$ decreases, in agreement with the asymptotic quasi-steady prediction of (4.7).

While the mode corresponding to the branch $B 1$ is the most unstable for large $m^{*}$, the situation changes dramatically for small mass ratios. Indeed, the two branches cross at $m^{*}=1.22, R e=44.35$. For smaller $m^{*}$, the instability occurs along the branch $B 2$. The corresponding threshold drops sharply as $m^{*}$ decreases, reaching $R e \approx 34.77$ in the limit $m^{*} \approx 0$. This switch from the $B 1$ branch to the $B 2$ one is associated with a jump in the Strouhal number, which is suddenly divided by a factor close to 2 .

The structure of a few eigenmodes is illustrated in figure 16; the corresponding values of the classification criteria are given in table 6. The first two (figure 16a,b) 


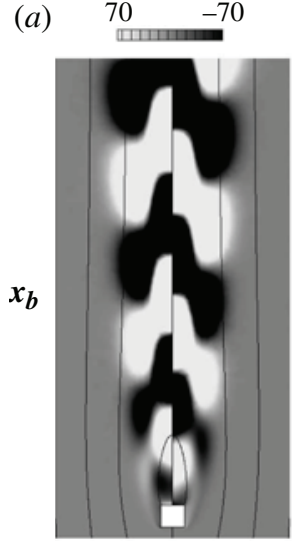

$y_{b}$ (b) 10

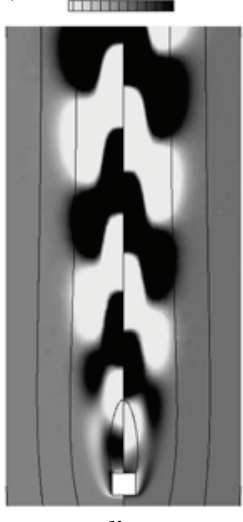

$y_{b}$ (c) $0.8 \quad-0.8$

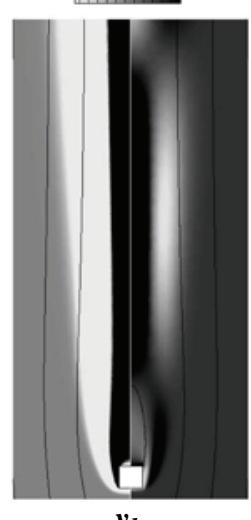

$y_{b}$ (d) $\quad \begin{array}{lll}2 & -2\end{array}$

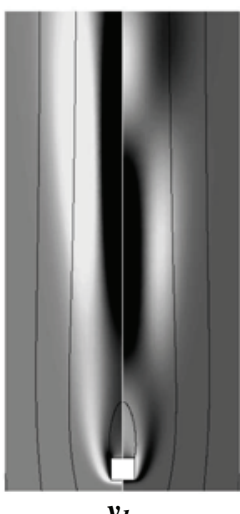

$y_{b}$

FIGURE 16. Structure of some eigenmodes of a square rod: $(a)$ mode $M_{1}$ with $m^{*}=10, \operatorname{Re}=$ 45; (b) mode $M_{2}$ with $m^{*}=1.22, R e=44.35$; (c) mode $M_{2}^{\prime}$ with $m^{*}=1.22, R e=44.35$; and (d) mode $M_{3}$ with $m^{*}=0.2, R e=37$. The modes are displayed with iso-levels of the vorticity component, using the same conventions as in figure 5.

\begin{tabular}{lccccccccc}
\hline$m^{*}$ & Re & $\begin{array}{c}\text { Mode } \\
\text { label }\end{array}$ & St & $\begin{array}{c}|\hat{\boldsymbol{\omega}} \cdot z| \\
(1.5,0)\end{array}$ & $\kappa$ & $|\hat{\gamma}|$ & $\phi_{\hat{\gamma}}$ & $|\hat{\alpha}|$ & $\phi_{\hat{\alpha}}$ \\
10 & 45 & $M_{1}$ & 0.105 & 1.3179 & 562 & 1.960 & $-\pi / 2.5$ & 1.901 & $-\pi / 2.3$ \\
1.22 & 44.35 & $M_{2}$ & 0.104 & 1.302 & 261 & 1.16 & $-\pi / 1.8$ & 1.22 & $-\pi / 4.2$ \\
1.22 & 44.35 & $M_{2}^{\prime}$ & 0.049 & 0.621 & 18.2 & 0.588 & $-\pi / 16$ & 0.438 & $-\pi / 12$ \\
0.2 & 37 & $M_{3}$ & 0.052 & 0.6450 & 67.5 & 0.646 & $-\pi / 8.2$ & 0.467 & $-\pi / 5.8$ \\
0.01 & 34.77 & & 0.052 & 0.655 & 11760 & 0.652 & $-\pi / 7.2$ & 0.492 & $-\pi / 5.3$ \\
10 & 52 & & 0.04 & 0.5002 & 3.01 & 0.178 & $-\pi / 14.3$ & 0.827 & $-\pi / 67$ \\
0.2 & 47 & & 0.105 & 1.3087 & 1062 & 0.803 & $-\pi / 2$ & 1.286 & $-\pi / 4.7$
\end{tabular}

TABLE 6. Values of various classification criteria for a selection of eigenmodes located close to the marginal stability curves in the case of a square rod. The nomenclature employed for the criteria is similar to that defined in table 3 and $\S 4.1 .3$. The labelled modes are displayed in figure 16.

are found along the branch $B 1$ and correspond to $m^{*}=10$ and to the crossing point $m^{*}=1.222$, respectively. Both of them have the visual appearance of 'fluid' modes of the VK1 type. Note that the maximum vorticity levels in the two panels of the figure suggest a weak coupling with the body motion. The last two modes (figure $16 c, d$ ) are found along the branch $B 2$ and correspond to the crossing point $m^{*}=1.222$ and to $m^{*}=0.2$, respectively. They display a rather different structure. The real part (left half of the two panels in the figure) exhibits a marked banded structure, which is the signature of a significant inclination of the body. The imaginary part (right half of panels) shows oscillations with a much larger wavelength than that of the previous two modes, a trend that is consistent with the much lower value of the Strouhal number.

Although we are unaware of any previous study with which the present findings for a square rod could be directly compared, it is worth mentioning that they exhibit strong qualitative similarities with the computational results reported by Alben (2008), who solved the coupled system of Navier-Stokes and Newton's equations for an 
elliptical rod of aspect ratio 3.4 and mass ratio $m^{*}=1.23$ (with present notation). Indeed, he found that, when the body is constrained to move vertically broadside on, the wake first becomes unstable at a critical Reynolds number $\sim 40$, while the lateral oscillations of the freely moving rod set in at a lower critical Reynolds number $R e_{c} \approx 23$. The periodic lateral motion then develops and the corresponding Strouhal number is $\sim 0.04$ for $R e=30$. This is in full qualitative agreement with what is observed here in the limit of small $\mathrm{m}^{*}$, where the critical Reynolds number is significantly lower than that associated with the 'fluid' mode and the corresponding Strouhal number is $\sim 0.05$. These similarities suggest that elliptical and rectangular shapes (and presumably other specific shapes) share a common scenario, namely, for small enough $m^{*}$ and $\chi$, the critical Reynolds number and the corresponding Strouhal number are associated with an 'aerodynamic' mode and are significantly smaller than those associated with the classical Von Kármán mode.

\section{Summary and concluding remarks}

The primary purpose of this work was to study the transition from a rectilinear path to an oscillating path for freely falling/rising bodies with a two-dimensional geometry. After deriving the linear equations governing the coupled fluid-body system, we investigated in detail two thin-body geometries, namely a quasi-infinitely thin plate and a rectangular plate with an aspect ratio $\chi=8$, both with a zero incidence angle in the base state, and a specific bluff-body geometry, namely a rod with a square cross-section.

Before considering arbitrary mass ratios, we paid particular attention to the limit case of very heavy bodies $\left(m^{*} \gg 1\right)$. For the three geometries under consideration (although results were only presented for the thin plate case), it turns out that in this limit the instability mechanism is similar to that of the wake of fixed bodies with the same geometry. In particular, the critical Reynolds number and the corresponding Strouhal number are identical to those found with a fixed body. Moreover, the components of the eigenmode corresponding to the body motion (i.e. its translational and angular velocities and its inclination) are asymptotically small in this limit. This means that for such heavy bodies the instability only manifests itself within the wake and has almost no signature on the path, which remains nearly vertical. This feature can be explained by noting that the unsteady loads (lift and torque) associated with the wake mode oscillate over a 'short' hydrodynamic time scale and can hardly excite any motion of the body, which, owing to its large inertia, evolves over a much longer time scale.

Although they have no effect on the leading instability mechanism, the additional degrees of freedom associated with the body displacements are responsible for the existence of four additional eigenmodes, which we refer to as 'aerodynamic' modes. These modes, which are characterized by much weaker eigenvalues, describe motions of the fluid-body system over a 'slow' time scale that is much longer than the hydrodynamic time scale over which the wake evolves. This slow evolution makes it possible to consider that, at leading order, the wake structure (and hence the loads) is entirely defined by the body kinematics. This view forms the basis of the 'quasi-static' model we developed in a recent paper, whose predictions agree well with the results of the present, global stability approach in the large- $m^{*}$ limit. In the range of Reynolds number for which the wake is known to be unstable, these additional modes are always damped in the case of thin bodies. In contrast, for a rod with a square section, two of these modes (which form a complex conjugate pair) become unstable for values 
of $R e$ not much higher than the threshold of the wake instability. For this reason, a 'rapid' vortex shedding mode and a 'slow' path oscillation are expected to exist simultaneously in a certain range of Reynolds number when such bluff bodies fall freely.

We then investigated the whole range of mass ratios and paid specific attention to $O(1)$ or smaller values of $m^{*}$. For the two thin bodies with $\chi=10^{4}$ and $\chi=8$, the limit $m^{*} \ll 1$ turned out to be qualitatively similar to that of large mass ratios. In particular, the critical Reynolds number and the corresponding Strouhal number are very close to the corresponding values in the opposite limit $m^{*} \gg 1$. Inspection of the eigenmode corresponding to destabilization in this low- $m^{*}$ range revealed that the 'fluid' component of the mode retains the same structure as in the fixed-body case, while the 'solid' component is small (although not asymptotically small). This trend indicates that, although the mass ratio is low, the instability still manifests itself through vortex shedding, with little effect of the body path. The case of intermediate mass ratios was found to be more complex. As $m^{*}$ decreases, we observed that the critical Reynolds number first decreases (down to $m^{*} \approx 4$ ), then increases (down to $m^{*} \approx 2$ ), before decreasing again. In this range of $m^{*}$, the 'solid' component of the eigenmodes is quite strong and comparable in magnitude to the 'fluid' component, making the instability associated with a significant displacement of the body. Nevertheless, the values of the Strouhal number remain comparable to those observed in the large- $m^{*}$ limit. Despite these general similarities, there is an important point of contrast between the two cases $\chi=10^{4}$ and $\chi=8$. Indeed, for the thinnest plate, a crossing between two branches of the marginal stability curve occurs for $m^{*}=1.66$. As $m^{*}$ crosses this threshold towards lower values, the Strouhal number abruptly decreases by $\sim 25 \%$ and the structure of the unstable mode that emerges changes from one with a significant body motion to one with almost no displacement of the body. Such a crossing does not exist in the case $\chi=8$. Nevertheless, the neutral curves indicate that there is a secondary mode that is only weakly damped for $m^{*} \approx 3$ and is likely to interact with the primary mode in this range of $m^{*}$.

The specific bluff-body geometry we considered, namely a rod with a square crosssection, revealed a different scenario. In this case two unstable modes with a different structure coexist whatever $m^{*}$ within the same range of $R e$. These two modes are the continuation of the 'fluid' and 'aerodynamic' modes identified in the large- $m^{*}$ limit. As the mass ratio decreases, these two modes were found to preserve their identity. Down to $m^{*}=1.22$, the 'fluid' mode remains the most amplified one while the 'aerodynamic' mode becomes the most amplified one for smaller $m^{*}$. This crossing between the two branches of the marginal stability curve is associated with an abrupt reduction of the Strouhal number by $\sim 50 \%$.

The present results provide new findings for contributing to the debate regarding the physical mechanisms responsible for path oscillations of buoyancy-driven bodies. In a forthcoming review (Ern et al. 2012), the available models and explanations for this class of phenomena are shown to belong to two general families. The first stream of explanations states that the dominant mechanism lies in the wake instability (vortex shedding), and that the body motion only plays a secondary role. The second series of approaches assumes that the whole picture is dominated by the role of the aerodynamic loads experienced by the body, regardless of any kind of intrinsic wake dynamics. Our results indicate that, for thin two-dimensional bodies, the mechanism responsible for the primary destabilization belongs to the first of these families. In particular, the transition occurs in the same range of Reynolds number $(\operatorname{Re} \approx 30)$ as in the wake of the corresponding fixed body; the two Strouhal 
numbers are also comparable. Interestingly, for the same class of thin bodies (falling cards), the fluttering/tumbling dynamics observed at much higher Reynolds number, say $R e=O\left(10^{3}\right)$, are more often explained using scenarios belonging to the second family. Indeed, 'aerodynamic' models in which the loads are modelled as a function of the body velocity, incidence angle, etc. are found to reproduce reasonably well the observed paths and transitions, provided the various empirical coefficients that they involve are suitably tuned (Pesavento \& Wang 2004; Andersen et al. 2005a). However, in the range of Re explored here, the 'quasi-static' model of FAM, which is a rigorous derivation of such an 'aerodynamic' model in the large- $m^{*}$ limit, fails to predict any instability of thin bodies. We thus have to conclude that the mechanisms responsible for unsteadiness and path instability are quite different in the range $R e \approx 30$ corresponding to the primary destabilization and in the range $R e=O\left(10^{3}\right)$ corresponding to large-amplitude fluttering and transition to tumbling. For this reason, it is highly desirable that future studies investigate the gap between these two wellseparated regimes and clarify the origin of this apparent paradox.

\section{Acknowledgements}

This work was supported by the French Research Agency (Agence Nationale de la Recherche) under grant ANR-09-BLAN-0132. The early development of the global stability code owes much to Denis Sipp and Philippe Meliga, who are warmly acknowledged. We also acknowledge Franck Auguste for fruitful discussions and for constantly opposing numerical counter-examples to any general rule we tried to elaborate (!), François Rincon for his assistance with the SLEPc library, and Olivier Chabiron and Patricia Ern for sharing their preliminary experimental results with us.

\section{REFERENCES}

Alben, S. 2008 An implicit method for coupled flow-body dynamics. J. Comput. Phys. 227, 4912-4933.

Andersen, A., Pesavento, U. \& Wang, Z. J. 2005 a Analysis of transitions between fluttering tumbling and steady descent of falling cards. J. Fluid Mech. 541, 91-104.

Andersen, A., Pesavento, U. \& WAng, Z. J. 2005 $b$ Unsteady aerodynamics of fluttering and tumbling plates. J. Fluid Mech. 541, 65-90.

Auguste, F. 2010 Instabilités de sillage générées derrière un corps solide cylindrique fixe ou mobile dans un fluide visqueux. PhD thesis, Université de Toulouse, France.

Auguste, F., Fabre, D. \& Magnaudet, J. 2009 Bifurcation in the wake of a thick circular disk. Theor. Comput. Fluid Dyn. 24, 305-313.

ERn, P., Risso, F., FABre, D. \& MAgnaudet, J. 2012 Wake-induced oscillatory paths of bodies freely rising or falling in fluids. Annu. Rev. Fluid Mech. 44, 97-121.

Fabre, D., Assemat, P. \& Magnaudet, J. 2011 A quasi-static approach to the stability of the path of heavy bodies falling within a viscous fluid. J. Fluids Struct. 27, 758-767.

Fabre, D., Auguste, F. \& Magnaudet, J. 2008 Bifurcation and symmetry breaking in the wake of axisymmetric bodies. Phys. Fluids 20, 051702.

JACKSON, C. P. 1987 A finite-element study of the onset of vortex shedding in flow past variously shaped bodies. J. Fluid Mech. 182, 23-45.

Jenny, M., Dusek, J. \& Bouchet, G. 2004 Instabilities and transition of a sphere falling or ascending freely in a Newtonian fluid. J. Fluid Mech. 508, 201-239.

JIN, C. \& XU, K. 2008 Numerical study of the unsteady aerodynamics of the freely falling plates. Commun. Comput. Phys. 3, 834-851.

KolomenskiY, D. \& SCHNEIDER, K. 2010 Numerical simulations of falling leaves using a pseudo-spectral method with volume penalization. Theor. Comput. Fluid Dyn. 24, 169-173. 
Lankadasu, A. \& Vengadesan, S. 2008 Onset of vortex shedding in planar shear flow past a square cylinder. Intl J. Heat Fluid Flow 29, 1054-1059.

Meliga, P., Chomaz, J.-M. \& Sipp, D. 2009 Global mode interaction and pattern selection in the wake of a disk: a weakly nonlinear expansion. J. Fluid Mech. 633, 159-189.

NAtARAJAN, R. \& Acrivos, A. 1993 The instability of the steady flow past spheres and disks. $J$. Fluid Mech. 254, 323-344.

Pesavento, U. \& WAng, Z. J. 2004 Falling paper: Navier-Stokes solutions, model of fluid forces, and centre of mass elevation. Phys. Rev. Lett. 93, 144501.

SAHA, A. K. 2007 Far-wake characteristics of two-dimensional flow past a normal flat plate. Phys. Fluids 19, 128110.

Sipp, D. \& LEBEDEV, A. 2007 Global stability of base and mean flows: a general approach and its applications to cylinder and open cavity flows. J. Fluid Mech. 593, 333-358.

Smith, E. H. 1971 Autorotating wings: an experimental investigation. J. Fluid Mech. 50, 513-534.

SOHANKAR, A., Norberg, C. \& DAVIDSON, L. 1998 Low-Reynolds-number flow around a square cylinder at incidence: study of blockage, onset of vortex shedding and outlet boundary condition. Intl J. Numer. Meth. Fluids 26, 39-56.

Thompson, M. C., Hourigan, K., Ryan, K. \& Sheard, G. 2006 Wake transition of two-dimensional cylinders and axisymmetric bluff bodies. J. Fluids Struct. 22, 793-806. 